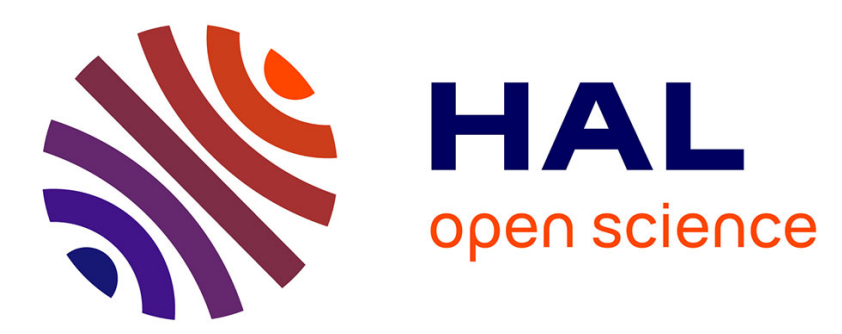

\title{
Milk protein and Oil-red-O staining of alveolar macrophages in chronic respiratory disease of infancy.
}

Frans de Baets, Claudia Aarts, Filomeen Haerynck, Sabine van Daele, Elke de Wachter, Iris de Schutter, Anne Malfroot, Petra Schelstraete

\section{- To cite this version:}

Frans de Baets, Claudia Aarts, Filomeen Haerynck, Sabine van Daele, Elke de Wachter, et al.. Milk protein and Oil-red-O staining of alveolar macrophages in chronic respiratory disease of infancy.. Pediatric Pulmonology, 2010, 45 (12), pp.1213. 10.1002/ppul.21310 . hal-00566724

\section{HAL Id: hal-00566724 \\ https://hal.science/hal-00566724}

Submitted on 17 Feb 2011

HAL is a multi-disciplinary open access archive for the deposit and dissemination of scientific research documents, whether they are published or not. The documents may come from teaching and research institutions in France or abroad, or from public or private research centers.
L'archive ouverte pluridisciplinaire HAL, est destinée au dépôt et à la diffusion de documents scientifiques de niveau recherche, publiés ou non, émanant des établissements d'enseignement et de recherche français ou étrangers, des laboratoires publics ou privés. 


\section{Milk protein and Oil-red-O staining of alveolar macrophages in chronic respiratory disease of infancy.}

\begin{tabular}{|r|l|}
\hline Journal: & Pediatric Pulmonology \\
\hline Manuscript ID: & PPUL-10-0041.R1 \\
\hline Wiley - Manuscript type: & Original Article \\
\hline Date Submitted by the & 09-May-2010 \\
\hline Complete List of Authors: & $\begin{array}{l}\text { De Baets, Frans; University Hospital Ghent, Paediatric Pulmonology } \\
\text { Aarts, Claudia; UMC St. Radboud Hospital, pediatric pulmonology } \\
\text { Haerynck, Filomeen; University Hospital Ghent, Department of } \\
\text { Paediatric Pulmonology } \\
\text { Van daele, Sabine; University Hospital Ghent, Department of } \\
\text { Paediatric Pulmonology } \\
\text { De Wachter, Elke; University Hospital Brussels, Pediatric } \\
\text { Pulmonology } \\
\text { De Schutter, Iris; University Hospital Brussels, Pediatric } \\
\text { Pulmonology } \\
\text { Malfroot, Anne; Universitair Ziekenhus Brussel, Pediatric } \\
\text { Respiratory Medicine, Cystic Fibrosis Clinic and Infectiology } \\
\text { Schelstraete, Petra; Ghent University Hospital, Pediatric } \\
\text { Pulmonology }\end{array}$ \\
\hline Keywords: & $\begin{array}{l}\text { aspiration, wheezing, infant, gastro-esophageal reflux, } \\
\text { laryngomalacia, tracheomalacia, Oil-Red-O staining }\end{array}$ \\
\hline \hline
\end{tabular}

\section{S ScholarONE" \\ Manuscript Central}


Milk protein staining of alveolar macrophages in chronic respiratory disease of infancy.

\section{Frans De Baets ${ }^{1}$, Claudia Aarts $^{2}$, Sabine Van daele ${ }^{1}$, Filomeen Haerynck ${ }^{1}$, Elke De} Wachter $^{3}$, Iris De Schutter ${ }^{3}$, Anne Malfroot ${ }^{3}$, Petra Schelstraete ${ }^{1}$

1. Department of Pediatric Pulmonology, Ghent University Hospital, Belgium.

2. Department of Pediatric Pulmonology UMC St. Radboud Hospital Nijmegen, The Netherlands

3. Department of Pediatric Pulmonology, University Hospital Brussels, Belgium

Keywords: aspiration, wheezing, infants, gastro-esophageal reflux, laryngomalacia, tracheomalacia 


\begin{abstract}
Aspiration is a suspected cause of chronic respiratory disease in infants.

We assessed the probability of aspiration by immunocytochemical staining for milk proteins ( $\alpha$-lactalbumin and $\beta$-lactoglobulin) in alveolar macrophages.

Broncho-alveolar lavage (BAL), 24-hour esophageal pH-measurement and/or gastroesophageal scintigraphy were performed in 111 children. Seventy nine patients were enrolled. Ten exclusive soya milk formula fed children served as a control group. Individual scores, expressed as the mean percentage of positive staining macrophages counted by 3 blinded authors were made. Relying on the control group, a positive score was defined as a score higher than $1 \%$.

A positive score was found in $26 \%$ (18/69). Forty four percent (8/11) of them had positive gastro-esophageal reflux (GER) tests. In 61\% (11/18) of positive staining patients a concomitant diagnosis of laryngo-/tracheomalacia was made. A positive score was found in $48 \%(11 / 23)$ of patients with laryngo-/ tracheomalacia, compared to $15 \%$ (7/46) in infants with normal laryngeal and tracheal anatomy.

Assuming the $1 \%$ criterion, persistent respiratory symptoms were associated with a positive immunostaining score, suggestive for aspiration, in $26 \%$, in $48 \%$ in case of concomitant laryngo- and/or tracheomalacia and in $15 \%$ of infants with normal laryngeal and tracheal anatomy.
\end{abstract}

\title{
INTRODUCTION
}

Recurrent pulmonary aspiration is a possible cause of chronic respiratory disease in children ${ }^{1-}$ 5 . Unfortunately, the diagnosis of aspiration is difficult to establish because a golden standard is lacking ${ }^{1-6}$. The currently available tests have either low sensitivity or low specifity .

Barium swallow during videofluoroscopy and gastroesophageal scintigraphy (milk-scan) have low sensitivity ${ }^{2,6,7}$. Recent studies have shown that calculation of the lipid-laden-alveolarmacrophage index (LLAM-index) carry very low sensitivity for aspiration: a high LLAMindex is found in children with respiratory disorders unrelated to aspiration. ${ }^{2,5}$.

Although up to 40-50\% of infants with gastro-esophageal reflux (GER) presents with respiratory symptoms ${ }^{7}$ the causal relationship between GER and respiratory symptoms remains obscure. Increased incidence of GER in children with respiratory symptoms is poorly understood and explained ${ }^{7,8}$.

Although laryngo- and tracheomalacia are not recorded as predisposing factors for aspiration in reference handbooks of pediatric respiratory disease, some scarce studies report an increased incidence of aspiration $^{9-11}$.

Research has been done on new diagnostic methods to study pulmonary aspiration ${ }^{12-, 14}$. Elidemir et al ${ }^{12}$ showed in a murine model that aspiration of milk could be diagnosed by immunocytochemical staining of the milk proteins, $\alpha$-lactalbumin and $\beta$-lactoglobulin, in alveolar macrophages recruited by broncho-alveolar lavage (BAL). Relying on this animal model Miller et $\mathrm{al}^{13}$ evaluated the feasibility of this diagnostic technique in clinical practice. They studied the immunocytochemical staining of milk proteins in tracheal aspirates from ventilated infants. We hypothesized that recurrent aspiration could be associated with persistent respiratory symptoms in infants and studied the incidence of positive immunocytochemical staining for milk proteins in alveolar macrophages of infants and young children with persistent respiratory symptoms, as a possible marker of aspiration.. 
A fiberbronchoscopy and BAL was done, using sedation and local anesthesia. The BAL was performed in the right lower lobe by instillation and aspiration of $3 \times 1 \mathrm{ml} / \mathrm{kg}$ body weight normal saline. BAL-fluid was used for cell differentiation and for the preparation of 6 cytospins. One of the 6 cytospins was stained with Giemsa for the white blood cell differentiation, the other 5 were fixed in acetone for 15 seconds on room temperature. The cytospins were kept away from sunlight immediately after processing.

Laryngomalacia was defined as a dynamic anomaly of the supraglottis causing stridor by narrowing of the laryngeal entry, tracheomalacia as an abnormal collapse $(>50 \%)$ of the trachea due to localized or generalized weakness of the tracheal wall, leading to respiratory obstruction.

Cytospin pellets were used for immunocytochemical staining for $\alpha$-lactalbumin and $\beta$ lactoglobulin.

The slides were fixed in acetone and blocked with 5\% PBS/BSA for 20 min, incubated with the blocking antibodies, Rabbit anti-human alpha-lactalbumin (catnr. L-0888, Sigma-Aldrich) and Rabbit anti-bovine lactoglobulin (catnr. A10-125, Bethyl Laboratories Inc.,ImTec Diagnostics NV), incubated with biotinylated anti-rabbit immunoglobulin and exposed to streptavidin-peroxidase and AEC chromogen. Slides were counterstained with hematoxylin. The slides were examined by 3 independent observers using light microscopy. Each one counted 100 consecutive macrophages and expressed the results in terms of percent positive cells. The mean percentage of the three observations was defined as the score. If there were less than 20 macrophages per slide, or if macrophage cell structure was distorted, the patient was excluded due to poor slide quality. The observers were blinded to the clinical status, formula feeding and GER diagnosis of the patients. The inter-observer coefficient of variation for scores above 10 , calculated as the mean of the individual percent difference to the mean score of the three observers, was $17 \%$. 
24-hour intra-esophageal $\mathrm{pH}$-measuring was performed in all patients. A positive test was defined relying on gastro-enterological criteria ${ }^{15,16}$. If the 24 -hour intra-esophageal $\mathrm{pH}$ measuring was normal, a gastroesophageal scintigraphy was performed to detect non-acid reflux ${ }^{17,18}$.

Statistical significance of differences between groups and subgroups for prevalence of positive GER tests or laryngo- and/or tracheomalacie was calculated using the $\chi^{2}$ test. The non-parametrical Wilcoxon test was used for calculation of statistical significance of differences in granulocyte counts between positive and negative staining patients. A p-value less than 0.05 was considered significant.

\section{RESULTS}

Fourty one percent (32/79) of patients had a positive 24-hour intra-esophageal $\mathrm{pH}$ measurement or a positive gastroesophageal scintigraphy.

In the control group, only in one child a $1 \%$ positive score was found for $\alpha$-lactalbumin, therefore in the study group all scores higher than $1 \%$ were considered positive. In 4 of the soya milk formula fed children a positive 24 -hour intra-esophageal $\mathrm{pH}$ monitoring or gastroesophageal scintigraphy was found, 3 children had tracheomalacia.

In $26 \%(18 / 69)$ of the cow milk formula fed children alveolar macrophages stained positively for milk proteins (12 for $\alpha$-lactalbumin and 6 for both $\alpha$-lactalbumin and $\beta$-lactoglobulin). The median score of positivity was $11 \%$ (range $2-72 \%$ ) (Table 1 ).

A bronchoscopic diagnosis of laryngo- and/or tracheomalacia was made in 33\% (23/69) of the cow milk formula fed infants. Immunocytochemical staining for milk proteins in this subgroup was positive in $48 \%(11 / 23)$, compared to $15 \%(7 / 46)(\mathrm{p}<0.004)$ in infants with normal laryngeal and tracheal anatomy, (Table 1, Fig 2, Fig 3A).

The prevalence of laryngo- or tracheomalacia in the positive immunostaining patients was $61 \%(11 / 18)$, compared to $24 \%(12 / 51)(\mathrm{p}<0.004)$ in negative staining patients (figure $3 \mathrm{~B})$.

Forty four percent (8/18) of patients with positive immunostaining had a positive GER test, compared to $39 \%(20 / 51)$ in negative staining patients (figure 3C, NS).

Forty eight percent (11/23) of all patients with laryngo- and/or tracheomalacie had a positive GER test, compared to 37\% (17/46) in infants with normal laryngeal and tracheal anatomy (NS). In the laryngo- and/or tracheomalacie group GER tests were positive for 36\% (4/11) in positive staining compared to $58 \%$ (7/12) in negative staining patients (Fig 3D, NS),

Fifty seven percent (4/7)of patients with a positive immunostaining and normal laryngeal and tracheal anatomy had a positive GER test, compared to 33\% (13/39) in the negative staining group (Fig3E, NS).

The median percentage of neutrophils in BAL-fluid from the 18 positively staining patients revealed to be $40,5 \%$ (range $17-77 \%$ ) compared to $21 \%$ (range 6-61\%) in the negative group (NS, $\mathrm{p}=0.11$, Wilcoxon test). 


\section{DISCUSSION}

In an experimental murine model Elidemir et al. ${ }^{13}$ reported a sensitive and specific test to detect aspiration of milk using immunocytochemical staining of $\alpha$-lactalbumine and $\beta$ lactoglobulin in alveolar macrophages. Immunocytochemical staining for $\alpha$-lactalbumine was positive 2 hours after the aspiration moment and remained positive until 96 hours later. No immunoreactivity for milkproteins was found in alveolar macrophages obtained from control mice or mice infected with RSV or Mycoplasma pneumoniae, emphasizing the specificity of the monoclonal antibodies. The same research group ${ }^{12}$ published a pilot study, using the immunocytochemical detection technique on milk proteins in tracheal aspirates of ventilated infants. Twelve percent (4/34 patients) of the control group however did show a positive staining for milk proteins before the introduction of any enteral feeding. We used infants on a soya milk formula diet as a control group. Out of these 10 control children, counting three times 100 alveolar macrophages per patient, only in one patient a $1 \%$ score was found. In the cow milk formula fed group only 4 patients (4/69) did show a $1 \%$ score. Therefore any score higher than $1 \%$ was considered positive.

Assuming this $1 \%$ criterion our study demonstrates a positive score in $26 \%$ of the study population, suggesting pulmonary aspiration.

Only in $44 \%$ of these patients the positive immunocytochemical staining score was corroborated by a positive GER test. No significant differences in prevalence of positive GER tests between negative and positive staining patients of any group or subgroup were found, precluding any association between positive scores and positive GER tests.

The positivity of the 24-hour intra-esophageal $\mathrm{pH}$-measurement was evaluated, relying on gastro-enterological criteria which are established to predict reflux esophagitis rather than to explain respiratory symptoms. Gorenstein et al reported that respiratory symptoms are not directly related to the severity of GER as measured by $\mathrm{pH}$ studies ${ }^{19}$ and positivity of a gastroesophageal scintigraphy relies only on a limited observation time.

Besides GER induced, aspiration in infants and young children can occur as a result of choking.

Recurrent aspiration into the respiratory tract is believed to be a common problem in children with predisposing anatomic, neuromuscular and functional disorders ${ }^{1}$.

In $61 \%$ of the positive staining patients a concomitant diagnose of laryngo- and/or tracheomalacia was made and a positive score was found in $48 \%$ of all infants with laryngoand/or tracheomalacia. These prevalence's of a positive score were significantly higher compared to children (15\%) with a normal laryngeal and tracheal anatomy, suggesting a higher probability of aspiration in infants with laryngo- and/or tracheomalacia. Laryngomalacia has been associated with GER in 50 to $80 \%$ of the cases ${ }^{20}$. We found positive GER tests in $48 \%$ of infants with laryngo- and/or tracheomalacia, a figure not significantly higher than in the group of children with persistent respiratory symptoms and normal laryngeal and tracheal anatomy.

Defining a true control group remains a difficult issue. From the murine model it was clear that positive immunocytochemical staining of alveolar macrophages for milk proteins is a sensitive and specific test to detect pulmonary aspiration ${ }^{13}$.

Assuming that cow milk proteins are not found in the human lungs, one should not expect to find positively staining alveolar macrophages. Miller et al. ${ }^{12}$ using rabbit anti-human $\alpha$ lactalbumin and rabbit anti-human $\beta$-lactoglobulin detected 12\% (3/34) positive staining in "true negatives" who did not receive any enteral feeding. The authors did not have a rational 
explanation for this finding. They suggest that cross-reaction of the anti-human antibodies to human lactalbumin and betaglobulin with structural proteins naturally existing in human airways may have occurred or that transamniotic passage of maternal dietary milk proteins into the lungs of newborns could not be excluded.

We used rabbit anti-human $\alpha$-lactalbumin and rabbit anti-bovine $\beta$-lactoglobulin, decreasing the possibility of cross reactivity at least for $\beta$-lactoglobuline. Because the median age of our patients was 9 months, transamniotic passage of maternal dietary milk proteins into the lungs is not applicable.

In order to create a golden standard an exclusive soyamilk formula fed aspirating group should be compared to an aspirating cow milk formula fed group to validate this technique in humans. Unfortunately this setting can hardly be reached.

A larger soya milk formula fed control group could have strengthened our findings.

Nevertheless relying on the prevalence of positive scores in our cow milk formula fed group, we would expect to detect at least 2 or 3 positive staining infants in the soya fed control group, representing 3 infants with tracheomalacia and 4 with a positive GER test.

Miller et al. ${ }^{12}$ did not use any quantification to define immunocytochemical staining positivity. One can imagine that minimal non specific binding of monoclonal antibodies to less qualitative BAL fluid samples, giving false positive results could have occurred. Quantification of the results by counting three times 100 macrophages and reporting the mean as a percentage of positivity could anticipate this problem and strengthens our results. The inter observer variability calculated by the coefficient of variation was acceptable for values above $10 \%$, but as expected larger for values between 2 and $9 \%$. Therefore the $1 \%$ cut off value defining a positive score can be argued, and further work using anti-bovine $\alpha$-lactalbumin and $\beta$-lactoglobulin is mandatory to strengthen or to redefine a cut off value. Intra-observer variability and for obvious reasons reproducibility of BAL specimens were not assessed.

This study confirms a high incidence of positive GER tests in children with persistent respiratory symptoms .

In positive staining samples, 12 were positive for $\alpha$-lactalbumin and 6 for both $\alpha$-lactalbumin and $\beta$-lactoglobulin, suggesting that immunocytochemical staining of $\alpha$-lactalbumin was more sensitive. In the study of Miller et al. ${ }^{12}$ only in 7 of the 12 children a combined $\alpha$-lactalbumin and $\beta$-lactoglobulin immunocytochemical staining was done, 6 patients were positive for $\beta$ lactoglobulin and 5 for $\alpha$-lactalbumin, 2 simultaneous for both.

In conclusion, assuming the $1 \%$ criterion of positivity, we found a positive immunocytochemical staining score for cow milk proteins in $26 \%$ of infants and young children with persistent respiratory symptoms, suggesting pulmonary aspiration. Compared to children with normal laryngeal and tracheal anatomy, children with laryngo- and/or tracheomalacia had significantly higher prevalence of positive scores. Positive staining patients had no statistically higher prevalence of positive GER tests. Further studies to strengthen or redefine a cut off value for positivity of the staining score are warranted 


\section{REFERENCES}

1. Bauer ML, Figueroa-Colon R, Georgeson K, Young DW. Chronic pulmonary aspiration in children. Southern Medical Journal, July 2003; Vol. 86, No. 7

2. Knauer-Fischer S, Ratjen F. Lipid-laden macrophages in bronchoalveolar lavage fluid as a marker for pulmonary aspiration. Pediatr Pulmonol. 1999;27:419-422

3. Bauer ML, Lyrene RK. Chronic aspiration in children: evaluation of the lipid-laden macrophage index. Pediatr Pulmonol. 1999;28:94-100

4. Ahrens P, Noll C, Kitz R, Willigens P, Zielen S, Hofmann D. Lipid-laden alveolar macrophage (LLAM): a useful marker of silent aspiration in children. Pediatr Pulmonol. 1999;28:83-88

5. Kazachkov M, Muhlenbach M, Livasy C, Noah T. Lipid laden macrophages indices and inflammation in BAL fluid of children with respiratory symptoms. ERJ 2001; 18: 790-795.

6. Sacco O, Fregonese B, Silvestri M, Sabatini F, Mattioli G, Rossi GA. Bronchoalveolar lavage and esophageal $\mathrm{pH}$ monitoring data in children with "difficult to treat" respiratory symptoms. Pediatr Pulmonol. 2000;30:313-319

7. Sheikh S, Stephen T, Howell L, Eid N. Gastroesophageal reflux in infants with wheezing. Pediatr Pulmonol. 1999;28:181-186

8. Vijayaratnam V, Lin CH, Simpson P, Tolia V. Lack of significant proximal esophageal acid reflux in infants presenting with respiratory symptoms. Pediatr Pulmonol 1999;27:231-235

9. Medula F, Guidi R, Tancredi G, Quantrucci S, Ratjen F, Bottero S, Vestiti K, Francalanci P, Cutrera R. Microapiration in infants with laryngomalacia. Laryngoscope 2004; 114: 1592-96

10. Dickson JM, Richter GT, Meinzen-Derr J, Rutter MJ, Thompson JM. Secondary airway lesions in children with laryngomalacia. Ann Oto Rhinol Laryngol 2009; 118: 37-43.

11. Yalcin E, Dogru D, Ozcelik U, Kiper N, Aslan AT, Gözaçan A. Tracheomalacia and bronchomalacia in 34 children: clinical and radiological profiles and associations with other diseases. Clin Ped 2005; 44: 777-81

12. Elidemir O, Fan LL, Colasurdo GN. A novel diagnostic method for pulmonary aspiration in a murine model: immunocytochemical staining of milk proteins in alveolar macrophages. Am J Respir Crit Care Med. 2000;161:622-626

13. Miller J, Colasurdo GN, Khan AM, Jajoo C, Patel TJ, Fan LL, Elidemir O. Immunocytochemical detection of milk proteins in tracheal aspirates of ventilated infants: a pilot study. Pediatric Pulmonol. 2002;34:369374

14. Farrell S, McMaster C, Gibson D, Shields MD, McCallion WA. Pepsin in bronchoalveolar lavage fluid: a specific and sensitive method of diagnosing gastro-oesophageal reflux-related pulmonary aspiration. Journal of Pediatric Surgery 2006;41:289-293

15. Vandenplas Y. Oesophageal $\mathrm{pH}$ monitoring for gastroesophagealreflux in infants and children. Chichester: John Wiley; 1992.

16. Pediatric GE reflux clinical practice guidelines. J. Pediatr Gastroenterol Nutr, Vol. 32, Suppl. 2, 2001

17. Piepsz. Recent advances in Pediatric Nuclear medicine. Sem Nucl Med 1995; 25: 165-182

18. Ravelli AM, Panarotto MB, Verdoni L, Consolati V, Bolognini S. Pulmonary aspiration shown by scintigraphy in gastroesophageal reflux-related respiratory disease. Chest 2006;130:1520-1526

19. Gorenstein A, Levine A, Boaz M, Mandelberg A, Serour F. Severity of acid gastroesophageal reflux assessed by pH metry: is it associated with respiratory disease? Pediatr Pulmonol. 2003;36:330-334

20. Bobin S, Attal P. Laryngotracheal manifestations of gastroesophagael reflux in children. Pediatr Pulmonol. 1999, suppl 18:73-75 
Table 1: Characteristics of positively staining children

\begin{tabular}{|c|c|c|c|c|c|}
\hline Gender & $\begin{array}{l}\text { age } \\
\text { (months) }\end{array}$ & bronchoscopy & pHmeasurement/scintigraphy & $\begin{array}{l}\text { score ALA } \\
(\%) \\
(\%)\end{array}$ & $\begin{array}{l}\text { score } B L G^{b} \\
(\%)\end{array}$ \\
\hline M & 6 & $\mathrm{nl}^{\mathrm{c}}$ & $\mathrm{nl}$ & 10 & 13 \\
\hline M & 10 & $\mathrm{nl}$ & $\mathrm{nl}$ & 7 & 0 \\
\hline M & 5 & larngo/tracheomalacie & $\mathrm{nl}$ & 7 & 0 \\
\hline $\mathrm{F}$ & 9,5 & laryngotracheomalacie & $\mathrm{nl}$ & 72 & 51 \\
\hline $\mathrm{F}$ & 8,5 & tracheomalacia & positive & 11 & 2 \\
\hline $\mathrm{F}$ & 4,5 & laryngotracheomalacie & $\mathrm{nl}$ & 13 & 0 \\
\hline $\mathrm{F}$ & 16 & $\mathrm{nl}$ & positive & 5 & 0 \\
\hline M & 9,5 & tracheomalacie & $\mathrm{nl}$ & 4 & 0 \\
\hline $\mathrm{F}$ & 14 & $\mathrm{nl}$ & $\mathrm{nl}$ & 2 & 0 \\
\hline $\mathrm{F}$ & 21 & $\mathrm{nl}$ & positive & 34 & 0 \\
\hline $\mathrm{F}$ & 4,5 & tracheomalacia & $\mathrm{nl}$ & 47 & 0 \\
\hline M & 8,5 & tracheomalacie & positive & 20 & 0 \\
\hline M & 10,5 & tracheomalacia & positive & 49 & 32 \\
\hline M & 7 & larngo/tracheomalacie & positive & 3 & 0 \\
\hline M & 5,5 & tracheomalacia & $\mathrm{nl}$ & 25 & 0 \\
\hline $\mathrm{F}$ & 11,5 & $\mathrm{nl}$ & positive & 48 & 22 \\
\hline M & 9 & $\mathrm{nl}$ & positive & 5 & 0 \\
\hline $\mathrm{F}$ & 4 & tracheomalacia & neg & 61 & 10 \\
\hline
\end{tabular}

a: $\alpha$-lactalbumin

b: $\beta$-lactoglobulin

c: normal 
Fig. 1:

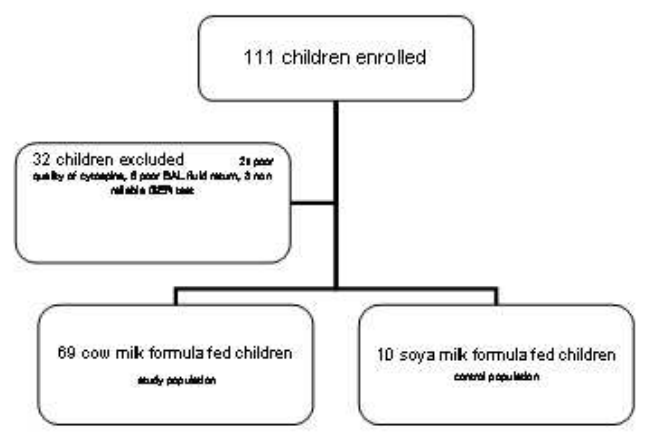

Study population 254x190mm (72 x 72 DPI)

John Wiley \& Sons, Inc. 


1
2
3
4
5
6
7
8
9
10
11
12
13
14
15
16
17
18
19
20
21
22
23
24
25
26
27
28
29
30
31
32
33
34
35
36
37
38
39
40
41
42
43
44
45
46
47
48
49
50
51
52
53
54
55
56
57
58
60

Fig. 2:

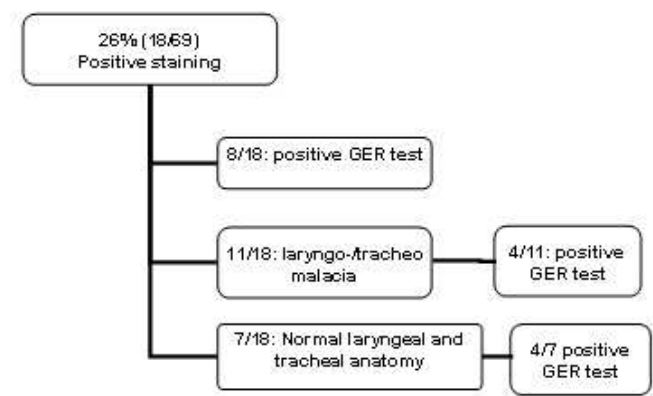

Concomitant findings in positive staining infants $254 \times 190 \mathrm{~mm}$ (72 x 72 DPI)

John Wiley \& Sons, Inc. 
Fig. 3:

A)

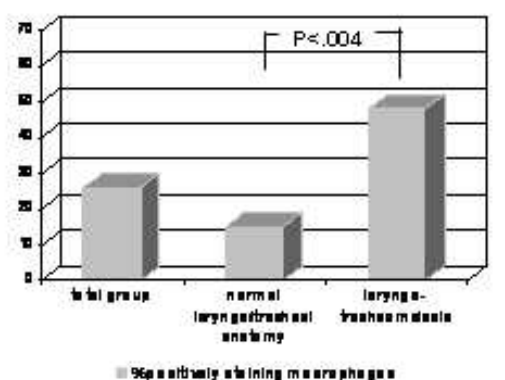

c)

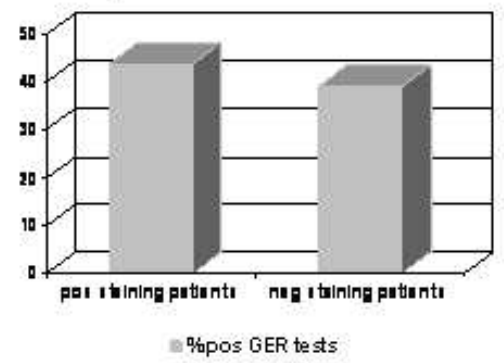

E)

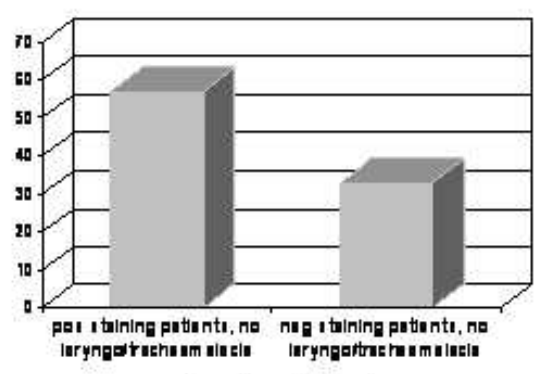

=\%positive GER tests
B)

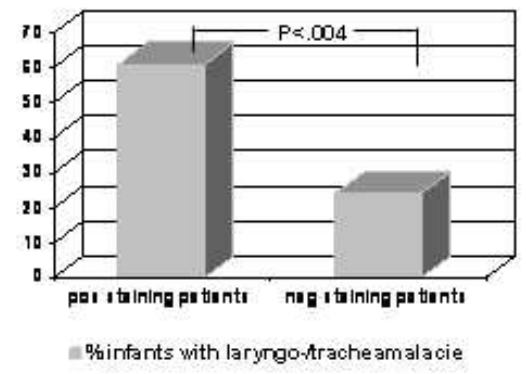

D)

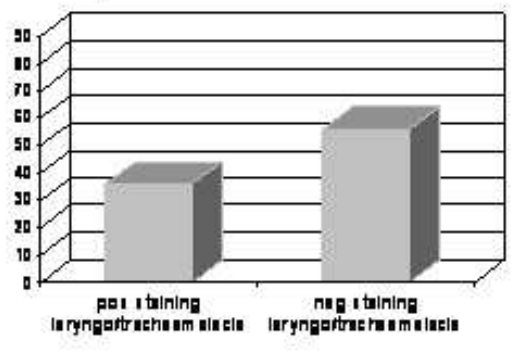

=\%positive GER tests

Comparison between groups and subgroups $190 \times 254 \mathrm{~mm}$ (72 x 72 DPI)

John Wiley \& Sons, Inc. 
Legends for figures.

Fig 1: Study population

Fig 2: Concomitant findings in positive staining infants 
Milk protein and Oil-red-O staining of alveolar macrophages in chronic respiratory disease of infancy.

Frans De Baets ${ }^{1}$, Claudia Aarts $^{2}$, Sabine Van daele ${ }^{1}$, Filomeen Haerynck ${ }^{1}$, Elke De Wachter $^{3}$, Iris De Schutter ${ }^{3}$, Anne Malfroot ${ }^{3}$, Petra Schelstraete ${ }^{1}$

1. Department of Pediatric Pulmonology, Ghent University Hospital, Belgium.

2. Department of Pediatric Pulmonology UMC St. Radboud Hospital Nijmegen, The Netherlands

3. Department of Pediatric Pulmonology, University Hospital Brussels, Belgium

Keywords: aspiration, wheezing, gastro-esophageal reflux, laryngomalacia, tracheomalacia, Oil-Red-O staining

Abbreviated title:

Assessment of aspiration in chronic respiratory disease 


\section{Summary}

Aspiration is a suspected cause of chronic respiratory disease in infants.

We assessed the probability of aspiration by immunocytochemical staining of alveolar macrophages for milk proteins ( $\alpha$-lactalbumin and $\beta$-lactoglobulin) and compared these findings with the Oil-Red-0 staining score.

Broncho-alveolar lavage (BAL), 24-hour esophageal pH-measurement and/or gastroesophageal scintigraphy were performed in 111 children. Seventy nine patients were enrolled. Ten exclusively soya milk formula fed children served as a control group. Individual scores, expressed as the mean percentage of positive staining macrophages counted by 3 blinded authors were made. Relying on the control group, a positive score was defined as a value higher than $1 \%$.

A positive score was found in $26 \%$ (18/69). Forty four percent $(8 / 18)$ of them had positive gastro-esophageal reflux (GER) tests. In 61\% (11/18) a concomitant diagnosis of laryngo/tracheomalacia was made. A positive score was found in $48 \%(11 / 23)$ of patients with laryngo-/ tracheomalacia, compared to $15 \%$ (7/46) in infants with normal laryngeal and tracheal anatomy.

No correlation was found between the immunocytochemical staining score for milk proteins and the Oil-Red-0 staining score.

We conclude that assuming the $1 \%$ criterion, persistent respiratory symptoms were associated with a positive immunostaining score, suggestive for aspiration, in $26 \%$ of infants, in $48 \%$ in case of concomitant laryngo- and/or tracheomalacia and in 15\% of infants with normal laryngeal and tracheal anatomy. No correlation was found between the immunocytochemical staining score for cow milk proteins and the Oil-Red-O staining score 


\section{INTRODUCTION}

Recurrent pulmonary aspiration is a possible cause of chronic respiratory disease in children ${ }^{1-}$

5 . Unfortunately, the diagnosis of aspiration is difficult to establish because a golden standard is lacking ${ }^{1-6}$. The currently available tests have either low sensitivity or low specifity. Barium swallowing studies during videofluoroscopy and gastroesophageal scintigraphy (milk-scan) have low sensitivity ${ }^{2,6,7}$. Recent studies have shown that calculation of the lipid-ladenalveolar-macrophage index (LLAM-index) carry very low sensitivity for aspiration: a high LLAM-index is found in children with respiratory disorders unrelated to aspiration. ${ }^{2,5}$. Although up to 40-50\% of infants with gastro-esophageal reflux (GER) presents with respiratory symptoms ${ }^{7}$ the causal relationship between GER and respiratory symptoms remains obscure. Increased incidence of GER in children with respiratory symptoms is poorly understood and explained ${ }^{7,8}$.

Although laryngo- and tracheomalacia are not recorded as predisposing factors for aspiration in reference handbooks of pediatric respiratory disease, some scarce studies report an increased incidence of aspiration $^{9-11}$.

Research has been done on new diagnostic methods to study pulmonary aspiration ${ }^{12-, 14}$. Elidemir et al ${ }^{12}$ showed in a murine model that aspiration of milk could be diagnosed by immunocytochemical staining for milk proteins, $\alpha$-lactalbumin and $\beta$-lactoglobulin, in alveolar macrophages, recruited by broncho-alveolar lavage (BAL). Relying on this animal model Miller et $\mathrm{al}^{13}$ evaluated the feasibility of this diagnostic technique in clinical practice. They studied the immunocytochemical staining of milk proteins in tracheal aspirates from ventilated infants.

We hypothesized that recurrent aspiration could be associated with persistent respiratory symptoms in infants and studied the incidence of positive immunocytochemical staining for milk proteins in alveolar macrophages of infants and young children with persistent respiratory symptoms, as a possible marker of aspiration.. 


\section{MATERIALS AND METHODS}

Patients were recruited in the pediatric pulmonary department of the University Hospitals of Ghent and Brussels, Belgium between January 2006 and August 2008. Infants and young children between 0 and 3 years who had persistent respiratory symptoms (wheezing and/or wed cough) unresponsive to regular asthma treatment (inhalation of $200 \mu \mathrm{g}$ fluticasone dipropionate or $400 \mu \mathrm{g}$ budesonide during one month) were evaluated. Only children with daily symptoms for at least one month were enrolled. In all patients a sweat test was done to exclude cystic fibrosis and relevant clinical information was obtained. Patients with obvious predisposing conditions to aspiration syndromes were withdrawn: neuromuscular and genetic diseases, achalasia, gastro-intestinal tract obstruction, micrognathia, retrognathia, macroglossia, tracheotomy.

During a 2,5 year-period, 111 children were evaluated. Seventy nine children (38 boys, median age 9 months, range 1-30.5 months) could be enrolled. Ten ( 5 boys, median age 11 months, range 8-15) of these 79 children were on an exclusively Soya milk formula diet and were considered as a control group. Thirty two patients were excluded: 21 because of poor quality of slides (poor staining quality, too much cell destruction), 8 because not enough BAL fluid was recovered and 3 children because neither a technically reliable 24-hour intraesophageal pH-monitoring nor a gastroesophageal scintigraphy were obtained (Fig 1). The study was approved by the Ethical Committees of the Ghent and Brussels University Hospital. Written informed consent was obtained from both parents.

Fiberbronchoscopy and BAL were done, using sedation and local anesthesia. The BAL was performed in the right lower lobe by instillation and aspiration of 3 times $1 \mathrm{ml} / \mathrm{kg}$ body weight normal saline. After each instillation the recovered fluid was saved in different recipients. The first sample was used for bacterial culture, the second for cell count, the last to make cell pellets by cytospin. A smear of one of the 6 cytospins was stained with Giemsa for white blood cell differentiation, smears of the other 5 were fixed in acetone for 15 seconds on room temperature. The slides were kept away from sunlight after processing.

Slides for Oil-O-Red staining were handled according to the protocol of Colombo et al ${ }^{15}$,: they were fixed in formaldehyde vapor for $5 \mathrm{~min}$, stained with Oil Red O for $20 \mathrm{~min}$, air-dried and counterstained with hematoxylin for 5 minutes. The LLAM-index was determined by 3 independent observers, blinded to the clinical status and GER diagnosis of the patients. The lipid content of 100 consecutive macrophages was scored on a scale of 0 to 4 ( 0 , not opacified; 1 , up to $1 / 4$ opacified; $2,1 / 4-1 / 2$ opacified; $3,1 / 2-3 / 4$ opacified; 4 , totally opacified) and the scores were summed. The possible range of values is from 0 to 400 . The inter observer variation coefficient, calculated as the mean of the individual difference to the mean score of the three observers, was 8 .

The slides for $\alpha$-lactalbumin and $\beta$-lactoglobulin immunocytochemical staining were fixed in acetone and blocked with 5\% PBS/BSA for $20 \mathrm{~min}$, incubated with the blocking antibodies, Rabbit anti-human alpha-lactalbumin (catnr. L-0888, Sigma-Aldrich) and Rabbit anti-bovine lactoglobulin (catnr. A10-125, Bethyl Laboratories Inc.,ImTec Diagnostics NV), incubated with biotinylated anti-rabbit immunoglobulin and exposed to streptavidin-peroxidase and AEC chromogen. Slides were counterstained with hematoxylin. The slides were examined using light microscopy by 3 independent observers, blinded to the clinical status, formula feeding and GER diagnosis of the patients. Each one counted 100 consecutive macrophages and expressed the results in terms of percent positive cells. The mean percentage of the three observations was defined as the score. If there were less than 20 macrophages per slide, or if 
macrophage cell structure was distorted, the patient was excluded due to poor slide quality. In the negative scored group, 43 patients were scored $0 \%$ by all three observers and in the remaining 8 negative patients only one observer scored positive with values ranging from 1 to $3 \%$. The inter-observer coefficient of variation, calculated as the mean of the individual difference to the mean score of the three observers, was 8 .

Laryngomalacia was defined as a dynamic anomaly of the supraglottis causing stridor by narrowing of the laryngeal entry, tracheomalacia as an abnormal collapse $(>50 \%)$ of the trachea due to localized or generalized weakness of the tracheal wall, leading to respiratory obstruction.

24-hour intra-esophageal $\mathrm{pH}$-measuring was performed in all patients. A positive test was defined relying on gastro-enterological criteria ${ }^{16,17}$. If the 24 -hour intra-esophageal $\mathrm{pH}$ measuring was normal, a gastroesophageal scintigraphy was performed to detect non-acid reflux ${ }^{18,19}$.

\section{Statistics}

Statistical significance of differences between groups and subgroups for prevalence of positive GER tests or laryngo- and/or tracheomalacie was calculated using the $\chi^{2}$ test. The non-parametrical Wilcoxon test was used for calculation of statistical significance of differences in granulocyte count and O-Red-Oil staining score between patients with a positive and a negative immunocytochemical staining score for $\alpha$-lactalbumin. A p-value less than 0.05 was considered significant. 


\section{RESULTS}

Fourty one percent (32/79) of patients had a positive 24-hour intra-esophageal $\mathrm{pH}$ measurement or a positive gastroesophageal scintigraphy.

In the control group, only in one child a $1 \%$ positive score was found for $\alpha$-lactalbumin,, therefore in the study group all scores higher than $1 \%$ were considered positive. In 4 of the soya milk formula fed children a positive 24 -hour intra-esophageal $\mathrm{pH}$ measurement or gastroesophageal scintigraphy were found, 3 children had tracheomalacia.

In $26 \%$ (18/69) of the cow milk formula fed children alveolar macrophages stained positively for milk proteins (12 for $\alpha$-lactalbumin and 6 for both $\alpha$-lactalbumin and $\beta$-lactoglobulin). The median value of a positive score was $11 \%$ (range 2-72\%) (Table 1).

A bronchoscopic diagnosis of laryngo- and/or tracheomalacia was made in 33\% (23/69) of the cow milk formula fed infants. Immunocytochemical staining for milk proteins in this subgroup was positive in $48 \%(11 / 23)$, compared to $15 \%(7 / 46)(\mathrm{p}<0.004)$ in infants with normal laryngeal and tracheal anatomy, (Table 1, Fig 2, Fig 3A).

The prevalence of laryngo- or tracheomalacia in the positive immunostaining patients was $61 \%(11 / 18)$, compared to $24 \%(12 / 51)(\mathrm{p}<0.004)$ in negative staining patients (figure $3 \mathrm{~B})$.

Forty four percent (8/18) of patients with positive immunostaining had a positive GER test, compared to $39 \%(20 / 51)$ in negative staining patients (figure 3C, NS).

Forty eight percent (11/23) of all patients with laryngo- and/or tracheomalacie had a positive GER test, compared to 37\% (17/46) in infants with normal laryngeal and tracheal anatomy (NS). In the laryngo- and/or tracheomalacia group GER tests were positive for 36\% (4/11) in positive staining compared to $58 \%$ (7/12) in negative staining patients (Fig 3D, NS), Fifty seven percent (4/7) of patients with a positive immunostaining and normal laryngeal and tracheal anatomy had a positive GER test, compared to 33\% (13/39) in the negative staining group (Fig3E, NS).

No correlation was found between the Oil-red-O score and the immunocytochemical staining score for $\alpha$-lactalbumin (Fig 4). The mean value of the Oil-red-O score in patients with a positive immunocytochemical staining score for $\alpha$-lactalbumin was 14 , range $0-73$. A significant difference was observed in Oil-red-O score between patients with a positive and a negative immunocytochemical staining score for $\alpha$-lactalbumin $(2$, IQR $0,38-5$ versus 4 , IQR $1,23-17, \mathrm{p}<0.04)$

The median percentage of neutrophils in BAL-fluid from the 18 positively staining patients revealed to be $41 \%$ (range $17-77 \%$ ) compared to $21 \%$ (range 6-61\%) in the negative group $(\mathrm{NS}, \mathrm{p}=0.11)$. 


\section{DISCUSSION}

In an experimental murine model Elidemir et al. ${ }^{13}$ reported a sensitive and specific test to detect aspiration of milk using immunocytochemical staining of $\alpha$-lactalbumine and $\beta$ lactoglobulin in alveolar macrophages. Immunocytochemical staining for $\alpha$-lactalbumine was positive 2 hours after the aspiration moment and remained positive until 96 hours later. No immunoreactivity for milkproteins was found in alveolar macrophages obtained from control mice or mice infected with RSV or Mycoplasma pneumoniae, emphasizing the specificity of the monoclonal antibodies. The same research group ${ }^{12}$ published a pilot study, using the immunocytochemical detection technique for milk proteins in tracheal aspirates of ventilated infants. Twelve percent (4/34 patients) of the control group however did show a positive staining for milk proteins before the introduction of any enteral feeding.

We used infants on an exclusively soya milk formula diet as a control group. Out of these 10 control children, counting three times 100 alveolar macrophages per patient, only in one patient a $1 \%$ score was found. In the cow milk formula fed group only 4 patients (4/69) did show a $1 \%$ score. Therefore any score higher than $1 \%$ was considered positive.

Assuming this $1 \%$ criterion our study demonstrates a positive score in $26 \%$ of the study population, suggesting pulmonary aspiration.

Only in $44 \%$ of these patients the positive immunocytochemical staining score was corroborated by a positive GER test. No significant differences in prevalence of positive GER tests between negative and positive staining patients of any group or subgroup were found, precluding any association between positive scores and positive GER tests.

The positivity of the 24-hour intra-esophageal $\mathrm{pH}$-measurement was evaluated, relying on gastro-enterological criteria which are established to predict reflux esophagitis rather than to explain respiratory symptoms. Gorenstein et al. reported that respiratory symptoms are not directly related to the severity of GER as measured by $\mathrm{pH}$ studies ${ }^{19}$ and positivity of a gastroesophageal scintigraphy relies only on a limited observation time.

Besides GER, choking can induce aspiration in infants and young children.

Recurrent aspiration into the respiratory tract is believed to be a common problem in children with predisposing respiratory and gastroenterological anatomic anomalies, neuromuscular disease and functional swallowing disorders ${ }^{1}$.

In $61 \%$ of the positive staining patients a concomitant diagnose of laryngo- and/or tracheomalacia was made and a positive score was found in $48 \%$ of all infants with laryngoand/or tracheomalacia. These prevalence's of a positive score were significantly higher compared to the score of children with a normal laryngeal and tracheal anatomy(15\%), suggesting a higher probability of aspiration in infants with laryngo- and/or tracheomalacia. Laryngomalacia has been associated with GER in 50 to $80 \%$ of the children ${ }^{21}$. We found positive GER tests in $48 \%$ of infants with laryngo- and/or tracheomalacia, a prevalence not significantly higher than in the group of children with persistent respiratory symptoms and normal laryngeal and tracheal anatomy.

Defining a true control group remains a difficult issue. From the murine model it was clear that positive immunocytochemical staining of alveolar macrophages for milk proteins is a sensitive and specific test to detect pulmonary aspiration ${ }^{13}$.

Assuming that cow milk proteins are not found in the human lungs, one should not expect to find positively staining alveolar macrophages. Miller et al. ${ }^{12}$ using rabbit anti-human $\alpha$ lactalbumin and rabbit anti-human $\beta$-lactoglobulin detected 12\% (3/34) positive staining in "true negatives" who did not receive any enteral feeding. The authors did not have a rational 
explanation for this finding. They suggest that cross-reaction of the anti-human antibodies to human lactalbumin and betaglobulin with structural proteins naturally existing in human airways may have occurred or that transamniotic passage of maternal dietary milk proteins into the lungs of newborns could not be excluded.

We used rabbit anti-human $\alpha$-lactalbumin and rabbit anti-bovine $\beta$-lactoglobulin, decreasing the possibility of cross reactivity at least for $\beta$-lactoglobuline. Because the median age of our patients was 9 months, transamniotic passage of maternal dietary milk proteins into the lungs is not applicable.

In order to create a golden standard an exclusively soyamilk formula fed aspirating group should be compared to an aspirating cow milk formula fed group to validate this technique in humans. Unfortunately this setting can hardly be reached.

A larger soya milk formula fed control group could have strengthened our findings.

Nevertheless relying on the prevalence of positive scores in our cow milk formula fed group, we would expect to find 2 or 3 aspirating infants in the soya fed control group, representing 3 infants with tracheomalacia and 4 with a positive GER test. Nevertheless the $1 \%$ cut off value defining a positive score can be argued, and further study is mandatory to strengthen or to redefine a cut off value.

Miller et al. ${ }^{12}$ did not use any quantification to define immunocytochemical staining positivity. One can imagine that minimal non specific binding of monoclonal antibodies to less qualitative BAL fluid samples, giving false positive results could have occurred. Quantification of the results by counting three times 100 macrophages and reporting the mean as a percentage of positivity could anticipate this problem and strengthens our results. The inter observer concordance in the negative scored group was good whereas the inter observer variability calculated by the coefficient of variation was low.

Intra-observer variability and for obvious reasons reproducibility of BAL specimens were not assessed.

This study confirms a high incidence of positive GER tests in children with persistent respiratory symptoms.

In positive staining samples, 12 were positive for $\alpha$-lactalbumin and 6 for both $\alpha$-lactalbumin and $\beta$-lactoglobulin, suggesting that immunocytochemical staining of $\alpha$-lactalbumin was more sensitive. In the study of Miller et al. ${ }^{12}$ only in 7 of the 12 children a combined $\alpha$-lactalbumin and $\beta$-lactoglobulin immunocytochemical staining was done, 6 patients were positive for $\beta$ lactoglobulin and 5 for $\alpha$-lactalbumin, 2 simultaneous for both.

The lack of positive correlation between the immunocytochemical score for $\alpha$-lactalbumin and the Oil-Red-O score confirms earlier reports that calculation of LLAM-index carry very low sensitivity for pulmonary aspiration ${ }^{2}$. None of our patients reached a score of 90 , which was suggestive for recurrent aspiration according to the study of Colombo et $\mathrm{al}^{15}$. The mean value of the Oil-Red-O score was comparable to their findings in the non aspirator group with pulmonary disease but lower than the mean score in the aspirator group for whom the respiratory morbidity was not mentioned. The mean value of the Oil-Red-O score in our study group was lower than the mean score in children with pulmonary disease reported by KnauerFischer et $\mathrm{al}^{2}$. In the latter study children were older and had severe pulmonary disease for years compared to our group of young children with only chronic respiratory signs for some months.

The significantly higher Oil-Red-O score in children with a positive immunocytochemical score for $\alpha$-lactalbumin suggests ongoing respiratory injury by chronic aspiration. 
In conclusion, assuming the $1 \%$ criterion of positivity, we found a positive immunocytochemical staining score for cow milk proteins in $26 \%$ of infants and young children with persistent respiratory symptoms, suggesting pulmonary aspiration. Compared to children with normal laryngeal and tracheal anatomy, children with laryngo- and/or tracheomalacia had a significantly higher prevalence of positive scores, suggesting an increased risk of aspiration. Positive staining patients had no statistically higher prevalence of positive GER tests. Further studies to strengthen or redefine a cut off value for positivity of the staining score are warranted. No correlation was found between the immunocytochemical staining score for cow milk proteins and the Oil-Red-O score confirming the low sensitivity of the latter test to detect aspiration. 
1. Bauer ML, Figueroa-Colon R, Georgeson K, Young DW. Chronic pulmonary aspiration in children. Southern Medical Journal, July 2003; Vol. 86, No. 7

2. Knauer-Fischer S, Ratjen F. Lipid-laden macrophages in bronchoalveolar lavage fluid as a marker for pulmonary aspiration. Pediatr Pulmonol. 1999;27:419-422

3. Bauer ML, Lyrene RK. Chronic aspiration in children: evaluation of the lipid-laden macrophage index. Pediatr Pulmonol. 1999;28:94-100

4. Ahrens P, Noll C, Kitz R, Willigens P, Zielen S, Hofmann D. Lipid-laden alveolar macrophage (LLAM): a useful marker of silent aspiration in children. Pediatr Pulmonol. 1999;28:83-88

5. Kazachkov M, Muhlenbach M, Livasy C, Noah T. Lipid laden macrophages indices and inflammation in BAL fluid of children with respiratory symptoms. ERJ 2001; 18: 790-795.

6. Sacco O, Fregonese B, Silvestri M, Sabatini F, Mattioli G, Rossi GA. Bronchoalveolar lavage and esophageal $\mathrm{pH}$ monitoring data in children with "difficult to treat" respiratory symptoms. Pediatr Pulmonol. 2000;30:313-319

7. Sheikh S, Stephen T, Howell L, Eid N. Gastroesophageal reflux in infants with wheezing. Pediatr Pulmonol. 1999;28:181-186

8. Vijayaratnam V, Lin CH, Simpson P, Tolia V. Lack of significant proximal esophageal acid reflux in infants presenting with respiratory symptoms. Pediatr Pulmonol 1999;27:231-235

9. Medula F, Guidi R, Tancredi G, Quantrucci S, Ratjen F, Bottero S, Vestiti K, Francalanci P, Cutrera R. Microapiration in infants with laryngomalacia. Laryngoscope 2004; 114: 1592-96

10. Dickson JM, Richter GT, Meinzen-Derr J, Rutter MJ, Thompson JM. Secondary airway lesions in children with laryngomalacia. Ann Oto Rhinol Laryngol 2009; 118: 37-43.

11. Yalcin E, Dogru D, Ozcelik U, Kiper N, Aslan AT, Gözaçan A. Tracheomalacia and bronchomalacia in 34 children: clinical and radiological profiles and associations with other diseases. Clin Ped 2005; 44: 777-81

12. Elidemir O, Fan LL, Colasurdo GN. A novel diagnostic method for pulmonary aspiration in a murine model: immunocytochemical staining of milk proteins in alveolar macrophages. Am J Respir Crit Care Med. 2000;161:622-626

13. Miller J, Colasurdo GN, Khan AM, Jajoo C, Patel TJ, Fan LL, Elidemir O. Immunocytochemical detection of milk proteins in tracheal aspirates of ventilated infants: a pilot study. Pediatric Pulmonol. 2002;34:369374

14. Farrell S, McMaster C, Gibson D, Shields MD, McCallion WA. Pepsin in bronchoalveolar lavage fluid: a specific and sensitive method of diagnosing gastro-oesophageal reflux-related pulmonary aspiration. Journal of Pediatric Surgery 2006;41:289-293

15. Colombo JL, Hallberg TK. Recurrent aspiration in children: lipid-laden alveolar macrophage quantitation. Pediatr Pulmonol 1987;3:86-89

16. Vandenplas Y. Oesophageal $\mathrm{pH}$ monitoring for gastroesophagealreflux in infants and children. Chichester: John Wiley; 1992.

17. Pediatric GE reflux clinical practice guidelines. J. Pediatr Gastroenterol Nutr, Vol. 32, Suppl. 2, 2001

18. Piepsz. Recent advances in Pediatric Nuclear medicine. Sem Nucl Med 1995; 25: 165-182

19. Ravelli AM, Panarotto MB, Verdoni L, Consolati V, Bolognini S. Pulmonary aspiration shown by scintigraphy in gastroesophageal reflux-related respiratory disease. Chest 2006;130:1520-1526

20. Gorenstein A, Levine A, Boaz M, Mandelberg A, Serour F. Severity of acid gastroesophageal reflux assessed by pH metry: is it associated with respiratory disease? Pediatr Pulmonol. 2003;36:330-334

21. Bobin S, Attal P. Laryngotracheal manifestations of gastroesophagael reflux in children. Pediatr Pulmonol. 1999, suppl 18:73-75 
Milk protein and Oil-red-O staining of alveolar macrophages in chronic respiratory disease of infancy.

Frans De Baets ${ }^{1}$, Claudia Aarts ${ }^{2}$, Sabine Van daele ${ }^{1}$, Filomeen Haerynck ${ }^{1}$, Elke De Formatted: English (U.K.) Wachter $^{3}$, Iris De Schutter ${ }^{3}$, Anne Malfroot ${ }^{3}$, Petra Schelstraete ${ }^{1}$

1. Department of Pediatric Pulmonology, Ghent University Hospital, Belgium.

Netherlands

3. Department of Pediatric Pulmonology, University Hospital Brussels, Belgium

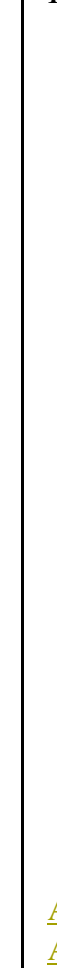

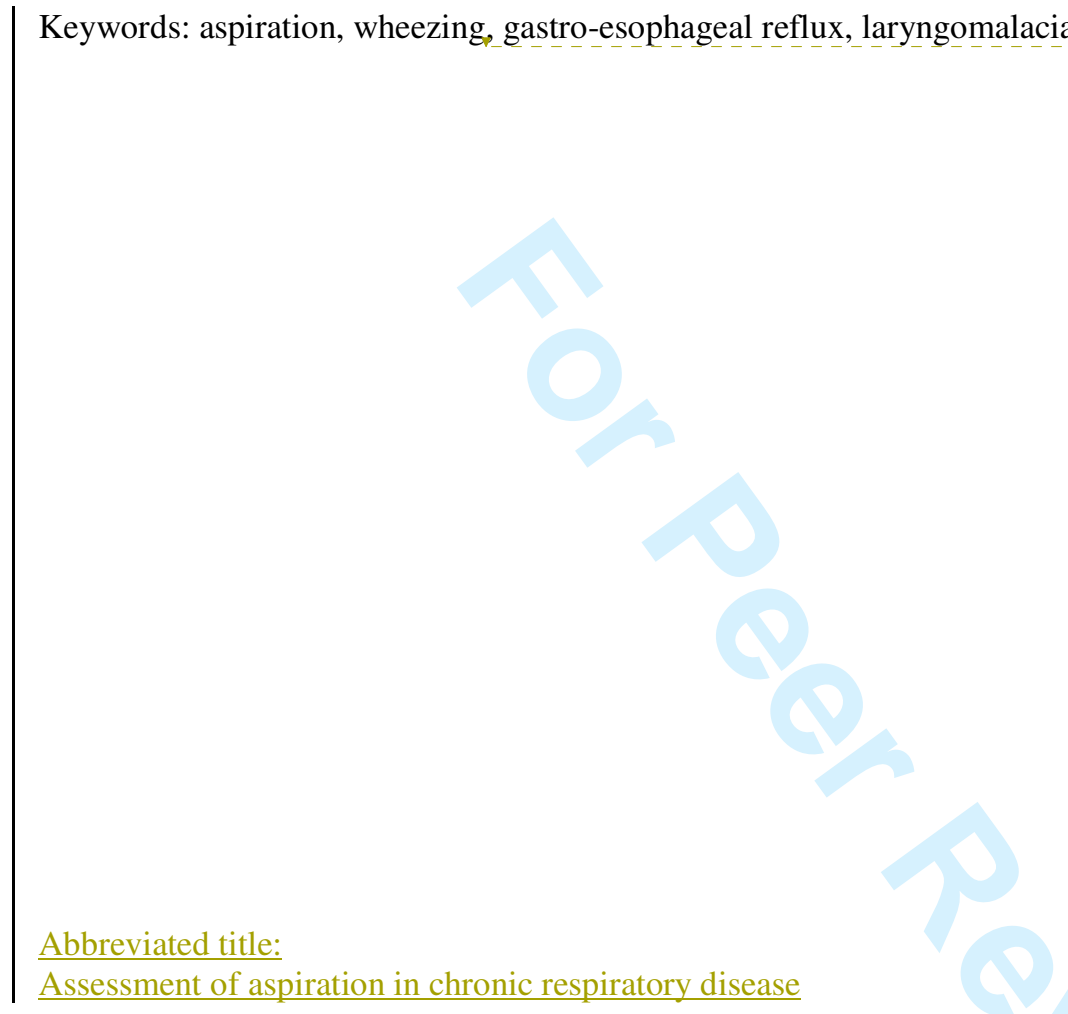


Summary

Aspiration is a suspected cause of chronic respiratory disease in infants.

We assessed the probability of aspiration by immunocytochemical staining of alveolar macrophages for milk proteins ( $\alpha$-lactalbumin and $\beta$-lactoglobulin) and compared these

findings with the Oil-Red-0 staining score.

Broncho-alveolar lavage (BAL), 24-hour esophageal pH-measurement and/or gastroesophageal scintigraphy were performed in 111 children. Seventy nine patients were enrolled. Ten exclusive soya milk formula fed children served as a control group. Individual scores, expressed as the mean percentage of positive staining macrophages counted by 3 blinded authors were made. Relying on the control group, a positive score was defined as a value, higher than $1 \%$.

A positive score was found in $26 \%$ (18/69). Forty four percent (8/18) of them had positive gastro-esophageal reflux (GER) tests. In $61 \%$ (11/18) a concomitant diagnosis of laryngo/tracheomalacia was made. A positive score was found in $48 \%$ (11/23) of patients with laryngo-/ tracheomalacia, compared to 15\% (7/46) in infants with normal laryngeal and tracheal anatomy.

No correlation was found between the immunocytochemical staining score for milk proteins and the Oil-Red-0 staining score.

We conclude that assuming the $1 \%$ criterion, persistent respiratory symptoms were associated with a positive immunostaining score, suggestive for aspiration, in $26 \%$ of infants, in $48 \%$ in case of concomitant laryngo- and/or tracheomalacia and in $15 \%$ of infants with normal laryngeal and tracheal anatomy. No correlation was found between the immunocytochemical staining score for cow milk proteins and the Oil-red-O score 


\section{INTRODUCTION}

Recurrent pulmonary aspiration is a possible cause of chronic respiratory disease in children ${ }^{1-}$

${ }^{5}$. Unfortunately, the diagnosis of aspiration is difficult to establish because a golden standard is lacking ${ }^{1-6}$. The currently available tests have either low sensitivity or low specifity .

Barium swallow during videofluoroscopy and gastroesophageal scintigraphy (milk-scan) have low sensitivity ${ }^{2,6,7}$. Recent studies have shown that calculation of the lipid-laden-alveolarmacrophage index (LLAM-index) carry very low sensitivity for aspiration: a high LLAMindex is found in children with respiratory disorders unrelated to aspiration. ${ }^{2,5}$.

Although up to 40-50\% of infants with gastro-esophageal reflux (GER) presents with respiratory symptoms ${ }^{7}$ the causal relationship between GER and respiratory symptoms remains obscure. Increased incidence of GER in children with respiratory symptoms is poorly understood and explained ${ }^{7,8}$.

Although laryngo- and tracheomalacia are not recorded as predisposing factors for aspiration in reference handbooks of pediatric respiratory disease, some scarce studies report an increased incidence of aspiration ${ }^{9-11}$.

Research has been done on new diagnostic methods to study pulmonary aspiration ${ }^{12-14}$. Elidemir et $\mathrm{al}^{12}$ showed in a murine model that aspiration of milk could be diagnosed by immunocytochemical staining for milk proteins, $\alpha$-lactalbumin and $\beta$-lactoglobulin, in alveolar macrophages, recruited by broncho-alveolar lavage (BAL). Relying on this animal model Miller et $\mathrm{al}^{13}$ evaluated the feasibility of this diagnostic technique in clinical practice. They studied the immunocytochemical staining of milk proteins in tracheal aspirates from | ventilated infants.

We hypothesized that recurrent aspiration could be associated with persistent respiratory symptoms in infants and studied the incidence of positive immunocytochemical staining for milk proteins in alveolar macrophages of infants and young children with persistent respiratory symptoms, as a possible marker of aspiration.. 
Patients were recruited in the pediatric pulmonary department of the University Hospitals of Ghent and Brussels between January 2006 and August 2008. Infants and young children

| between 0 and 3 years who had persistent respiratory symptoms (wheezing and/or wed cough), unresponsive to regular asthma treatment (inhalation of $200 \mu \mathrm{g}$ fluticasone dipropionate or $400 \mu \mathrm{g}$ budesonide during one month) were evaluated. Only children with daily symptoms for at least one month were enrolled. In all patients a sweat test was done to exclude cystic fibrosis and relevant clinical information was obtained. Patients with obvious predisposing conditions to aspiration syndromes were withdrawn: neuromuscular and genetic diseases, achalasia, gastro-intestinal tract obstruction, micrognathia, retrognathia, macroglossia, tracheotomy.

During a 2,5 year-period, 111 children were evaluated. Seventy nine children (38 boys, median age 9 months, range 1-30.5 months) could be enrolled. Ten ( 5 boys, median age 11 months, range 8-15) of these 79 children were on an exclusive Soya milk formula diet and were considered as a control group. Thirty two patients were excluded: 21 because of poor

| quality of slides (poor staining quality, too much cell destruction), 8 because not enough BAL fluid was recovered and 3 children because neither a technically reliable 24 -hour intraesophageal pH-monitoring nor a gastroesophageal scintigraphy were obtained (Fig 1). The study was approved by the Ethical Committees of the Ghent and Brussels University Hospital. Written informed consent was obtained from both parents.

A fiberbronchoscopy and BAL was done, using sedation and local anesthesia. The BAL was performed in the right lower lobe by instillation and aspiration of $3 \times 1 \mathrm{ml} / \mathrm{kg}$ body weight normal saline. After each instillation the recovered fluid was saved in different recipients. The first sample was used for bacterial culture, the second for cell count, the last to make cell pellets by cytospin. A smear of one of the 6 cytospins was stained with Giemsa for white blood cell differentiation, smears of the other 5 were fixed in acetone for 15 seconds on room temperature. The slides, were kept away from sunlight immediately after processing.

Slides for Oil-O-Red staining were handled according to the protocol of Colombo et all 15 they were fixed in formaldehyde vapor for $5 \mathrm{~min}$, stained with Oil Red O for $20 \mathrm{~min}$, air-dried and counterstained with hematoxylin for 5 minutes. The LLAM-index was determined by 3 independent observers, blinded to the clinical status and GER diagnosis of the patients. The lipid content of 100 consecutive macrophages was scored on a scale of 0 to $4(0$, not opacified; 1 , up to $1 / 4$ opacified; $2,1 / 4-1 / 2$ opacified; $3,1 / 2-3 / 4$ opacified; 4 , totally opacified) and the scores were summed. The possible range of values is from 0 to 400 . The inter observer variation coefficient, calculated as the mean of the individual difference to the mean score of the three observers, was 8.

The slides for $\alpha$-lactalbumin and $\beta$-lactoglobulin immunocytochemical staining were fixed in acetone and blocked with 5\% PBS/BSA for 20 min, incubated with the blocking antibodies, Rabbit anti-human alpha-lactalbumin (catnr. L-0888, Sigma-Aldrich) and Rabbit anti-bovine lactoglobulin (catnr. A10-125, Bethyl Laboratories Inc.,ImTec Diagnostics NV), incubated with biotinylated anti-rabbit immunoglobulin and exposed to streptavidin-peroxidase and AEC chromogen. Slides were counterstained with hematoxylin. The slides were examined by 3 independent observers using light microscopy, blinded to the clinical status, formula feeding and GER diagnosis of the patients. Each one counted 100 consecutive macrophages and expressed the results in terms of percent positive cells. The mean percentage of the three observations was defined as the score. If there were less than 20 macrophages per slide, or if
Deleted: and/or cough

Deleted: bronchorrea)

Deleted: BAL specimens

Deleted: BAL-fluid was used for cell

differentiation and for the preparation of 6 cytospins. $\mathrm{O}$

Deleted: the

Deleted: cytospins

Deleted: Laryngomalacia was defined as a dynamic anomaly of the supraglottis causing stridor by narrowing of the laryngeal entry, tracheomalacia as an abnormal collapse $(>50 \%)$ of the trachea due to localized or generalized weakness of the tracheal wall, leading to respiratory obstruction. If

II

Cytospin pellets were used for immunocytochemical staining for $\alpha$ lactalbumin and $\beta$-lactoglobulin.

Formatted: Superscript

Deleted: The slides 
macrophage cell structure was distorted, the patient was excluded due to poor slide quality. In the negative scored group 43 patients were scored $0 \%$ by the three observers and in the remaining 8 negative patients only one observer scored positive with values ranging from 1 to $3 \%$. The inter-observer coefficient of variation, calculated as the mean of the indival difference to the mean score of the three observers, was 8 .

Laryngomalacia was defined as a dynamic anomaly of the supraglottis causing stridor by narrowing of the laryngeal entry, tracheomalacia as an abnormal collapse (>50\%) of the trachea due to localized or generalized weakness of the tracheal wall, leading to respiratory obstruction.

24-hour intra-esophageal $\mathrm{pH}$-measuring was performed in all patients. A positive test was | defined relying on gastro-enterological criteria 16,17 . If the 24-hour intra-esophageal $\mathrm{pH}-$ measuring was normal, a gastroesophageal scintigraphy was performed to detect non-acid | reflux 1,19 .

\section{Statistics}

Statistical significance of differences between groups and subgroups for prevalence of positive GER tests or laryngo- and/or tracheomalacie was calculated using the $\chi^{2}$ test. The non-parametrical Wilcoxon test was used for calculation of statistical significance of differences in granulocyte counts between positive and negative staining patients and difference in O-Red-Oil staining score between patients with a positive and a negative immunocytochemical staining score for $\alpha$-lactalbumin A p-value less than 0.05 was considered significant.

\begin{tabular}{l} 
Deleted: \\
Deleted: The observers were blinded to \\
the clinical status, formula feeding and \\
GER diagnosis of the patients. \\
Formatted: Font: Not Italic \\
Formatted: Font: Not Italic \\
\hline Formatted: Font: Not Italic \\
\hline Formatted: Font: Not Italic \\
Deleted: for scores above 10 \\
\hline Deleted: percent \\
\hline Deleted: $17 \%$ \\
\hline Deleted: ${ }^{5}$ \\
\hline Deleted: ${ }^{6}$ \\
\hline Deleted: ${ }^{7}$ \\
Deleted: ${ }^{8}$ \\
\hline Formatted: Font: Bold \\
\hline
\end{tabular}




\section{RESULTS}

Fourty one percent (32/79) of patients had a positive 24-hour intra-esophageal $\mathrm{pH}$ measurement or a positive gastroesophageal scintigraphy.

In the control group, only in one child a $1 \%$ positive score was found for $\alpha$-lactalbumin,, therefore in the study group all scores higher than $1 \%$ were considered positive. In 4 of the soya milk formula fed children a positive 24 -hour intra-esophageal $\mathrm{pH}$ measurement or gastroesophageal scintigraphy were found, 3 children had tracheomalacia.

In $26 \%$ (18/69) of the cow milk formula fed children alveolar macrophages stained positively for milk proteins (12 for $\alpha$-lactalbumin and 6 for both $\alpha$-lactalbumin and $\beta$-lactoglobulin). The | median value of a positive score was $11 \%$ (range 2-72\%) (Table 1).

A bronchoscopic diagnosis of laryngo- and/or tracheomalacia was made in $33 \%(23 / 69)$ of the cow milk formula fed infants. Immunocytochemical staining for milk proteins in this subgroup was positive in $48 \%(11 / 23)$, compared to $15 \%(7 / 46)(\mathrm{p}<0.004)$ in infants with normal laryngeal and tracheal anatomy, (Table 1, Fig 2, Fig 3A).

The prevalence of laryngo- or tracheomalacia in the positive immunostaining patients was $61 \%(11 / 18)$, compared to $24 \%(12 / 51)(\mathrm{p}<0.004)$ in negative staining patients (figure $3 \mathrm{~B})$.

Forty four percent (8/18) of patients with positive immunostaining had a positive GER test, compared to $39 \%$ (20/51) in negative staining patients (figure 3C, NS).

| Forty eight percent (11/23) of all patients with laryngo- and/or tracheomalacie had a positive GER test, compared to 37\% (17/46) in infants with normal laryngeal and tracheal anatomy

(NS). In the laryngo- and/or tracheomalacia group GER tests were positive for 36\% (4/11) in positive staining compared to $58 \%$ (7/12) in negative staining patients (Fig $3 \mathrm{D}, \mathrm{NS}$ ),

| Fifty seven percent (4/7) of patients with a positive immunostaining and normal laryngeal and tracheal anatomy had a positive GER test, compared to $33 \%(13 / 39)$ in the negative staining group (Fig3E, NS).

No correlation was found between the Oil-red-O score and the immunocytochemical staining score for $\alpha$-lactalbumin (Fig 4). The mean value of the Oil-red-O score in patients with a positive immunocytochemical staining score for $\alpha$-lactalbumin was 14 , range 0-73. A significant difference was observed in Oil-red-O score between patients with a positive and a negative immunocytochemical staining score for $\alpha$-lactalbumin $(2$, IQR $0,38-5$ versus $4,1 Q R$ $1,23-17, \mathrm{p}<0.04)$

The median percentage of neutrophils in BAL-fluid from the 18 positively staining patients revealed to be $41 \%$ (range $17-77 \%$ ) compared to $21 \%$ (range 6-61\%) in the negative group $(\mathrm{NS}, \mathrm{p}=0.11$ ).

Deleted: 0,5

Deleted: , Wilcoxon test 


\section{DISCUSSION}

In an experimental murine model Elidemir et al. ${ }^{13}$ reported a sensitive and specific test to detect aspiration of milk using immunocytochemical staining of $\alpha$-lactalbumine and $\beta$ lactoglobulin in alveolar macrophages. Immunocytochemical staining for $\alpha$-lactalbumine was positive 2 hours after the aspiration moment and remained positive until 96 hours later. No immunoreactivity for milkproteins was found in alveolar macrophages obtained from control mice or mice infected with RSV or Mycoplasma pneumoniae, emphasizing the specificity of the monoclonal antibodies. The same research group ${ }^{12}$ published a pilot study, using the

| immunocytochemical detection technique for milk proteins in tracheal aspirates of ventilated infants. Twelve percent (4/34 patients) of the control group however did show a positive staining for milk proteins before the introduction of any enteral feeding.

| We used infants on an exclusive soya milk formula diet as a control group. Out of these 10 control children, counting three times 100 alveolar macrophages per patient, only in one patient a $1 \%$ score was found. In the cow milk formula fed group only 4 patients (4/69) did show a $1 \%$ score. Therefore any score higher than $1 \%$ was considered positive.

Assuming this $1 \%$ criterion our study demonstrates a positive score in $26 \%$ of the study population, suggesting pulmonary aspiration.

Only in $44 \%$ of these patients the positive immunocytochemical staining score was corroborated by a positive GER test. No significant differences in prevalence of positive GER tests between negative and positive staining patients of any group or subgroup were found, precluding any association between positive scores and positive GER tests.

The positivity of the 24-hour intra-esophageal $\mathrm{pH}$-measurement was evaluated, relying on gastro-enterological criteria which are established to predict reflux esophagitis rather than to explain respiratory symptoms. Gorenstein et al reported that respiratory symptoms are not directly related to the severity of GER as measured by $\mathrm{pH}$ studies ${ }^{19}$ and positivity of a gastroesophageal scintigraphy relies only on a limited observation time.

Besides GER ${ }_{2}$ choking can induce aspiration in infants and young children. Recurrent aspiration into the respiratory tract is believed to be a common problem in children with predisposing respiratory and gastroenterological anatomic anomalies, neuromuscular disease and functional swallowing disorders ${ }^{1}$.

In $61 \%$ of the positive staining patients a concomitant diagnose of laryngo- and/or tracheomalacia was made and a positive score was found in $48 \%$ of all infants with laryngoand/or tracheomalacia. These prevalence's of a positive score were significantly higher | compared to the score of children with a normal laryngeal and tracheal anatomy $(15 \%)$, suggesting a higher probability of aspiration in infants with laryngo- and/or tracheomalacia. Laryngomalacia has been associated with GER in 50 to $80 \%$ of the children ${ }^{21}$. We found positive GER tests in $48 \%$ of infants with laryngo- and/or tracheomalacia, a prevalence not significantly higher than in the group of children with persistent respiratory symptoms and normal laryngeal and tracheal anatomy.

Defining a true control group remains a difficult issue. From the murine model it was clear that positive immunocytochemical staining of alveolar macrophages for milk proteins is a sensitive and specific test to detect pulmonary aspiration ${ }^{13}$.

Assuming that cow milk proteins are not found in the human lungs, one should not expect to find positively staining alveolar macrophages. Miller et al. ${ }^{12}$ using rabbit anti-human $\alpha$ lactalbumin and rabbit anti-human $\beta$-lactoglobulin detected $12 \%(3 / 34)$ positive staining in "true negatives" who did not receive any enteral feeding. The authors did not have a rational 
explanation for this finding. They suggest that cross-reaction of the anti-human antibodies to human lactalbumin and betaglobulin with structural proteins naturally existing in human airways may have occurred or that transamniotic passage of maternal dietary milk proteins into the lungs of newborns could not be excluded.

We used rabbit anti-human $\alpha$-lactalbumin and rabbit anti-bovine $\beta$-lactoglobulin, decreasing the possibility of cross reactivity at least for $\beta$-lactoglobuline. Because the median age of our patients was 9 months, transamniotic passage of maternal dietary milk proteins into the lungs is not applicable.

In order to create a golden standard an exclusive soyamilk formula fed aspirating group should be compared to an aspirating cow milk formula fed group to validate this technique in humans. Unfortunately this setting can hardly be reached.

A larger soya milk formula fed control group could have strengthened our findings.

Nevertheless relying on the prevalence of positive scores in our cow milk formula fed group, we would expect to detect at least 2 or 3 positive staining infants in the soya fed control group, representing 3 infants with tracheomalacia and 4 with a positive GER test. Nevertheless the $1 \%$ cut off value defining a positive score can be argued, and further study is mandatory to strengthen or to redefine a cut off value.

Miller et al. ${ }^{12}$ did not use any quantification to define immunocytochemical staining positivity. One can imagine that minimal non specific binding of monoclonal antibodies to less qualitative BAL fluid samples, giving false positive results could have occurred. Quantification of the results by counting three times 100 macrophages and reporting the mean as a percentage of positivity could anticipate this problem and strengthens our results.

The inter observer concordance in the negative scoring group was good whereas the inter observer variability calculated by the coefficient of variation was low

Intra-observer variability and for obvious reasons reproducibility of BAL specimens were not assessed.

This study confirms a high incidence of positive GER tests in children with persistent respiratory symptoms .

In positive staining samples, 12 were positive for $\alpha$-lactalbumin and 6 for both $\alpha$-lactalbumin and $\beta$-lactoglobulin, suggesting that immunocytochemical staining of $\alpha$-lactalbumin was more sensitive. In the study of Miller et al. ${ }^{12}$ only in 7 of the 12 children a combined $\alpha$-lactalbumin and $\beta$-lactoglobulin immunocytochemical staining was done, 6 patients were positive for $\beta$ lactoglobulin and 5 for $\alpha$-lactalbumin, 2 simultaneous for both.

The lack of positive correlation between the immunocytochemical score for $\alpha$-lactalbumin and the Oil-red-O score confirms earlier reports that calculation of LLAM-index carry very low sensitivity for pulmonary aspiration ${ }^{2}$. None of our patients reached a score of 90 , which was suggestive for recurrent aspiration according to the study of Colombo et $\mathrm{al}^{15}$. The mean value of the Oil-red-O score was comparable to their findings in the non aspirator group with pulmonary disease but lower than the mean score in the aspirator group for whom the respiratory morbidity was not mentioned. The mean value of the Oil-red-O score was lower than the mean score in children with pulmonary disease reported by Knauer-Fischer et $\mathrm{al}^{2}$. In the latter study children were older and had severe pulmonary disease for years compared to our group of young children with only chronic respiratory signs for some months. The significantly higher Oil-red-O score in children with a positive immunocytochemical score for $\alpha$-lactalbumin suggests ongoing respiratory injury by chronic aspiration.

\section{Deleted: $\mathrm{T}$}

\section{Formatted: Font: Not Italic}

Deleted: acceptable for values above $10 \%$, but as expected larger for values between 2 and $9 \%$

Deleted: Therefore the $1 \%$ cut off value defining a positive score can be argued, and further work using anti-bovine II

$\alpha$-lactalbumin and $\beta$-lactoglobulin is mandatory to strengthen or to redefine a cut off value.

Formatted: Superscript

Formatted: Superscript

Formatted: Font: $12 \mathrm{pt}$

Formatted: Superscript 
In conclusion, assuming the $1 \%$ criterion of positivity, we found a positive immunocytochemical staining score for cow milk proteins in $26 \%$ of infants and young children with persistent respiratory symptoms, suggesting pulmonary aspiration. Compared to children with normal laryngeal and tracheal anatomy, children with laryngo- and/or tracheomalacia had a significantly higher prevalence of positive scores, suggesting an increased risk of aspiration. Positive staining patients had no statistically higher prevalence of positive GER tests. Further studies to strengthen or redefine a cut off value for positivity of the staining score are warranted. No correlation was found between the immunocytochemical staining score for cow milk proteins and the Oil-red-O score confirming the low sensitivity of the latter test to detect aspiration. 
32 children excluded

21 poor quality of slides, 8 poor BAL

fluid return, 3 non reliable GER test

111 children enrolled

69 cow milk formula fed children

study population
10 soya milk formula fed

children

control population 
Table 1: Characteristics of positively staining children (ALA: $\alpha$-lactalbumin, BLG: $\beta$ -

Formatted: Font: Bold lactoglobulin, ORO: Oil-Red-O, nl: normal)

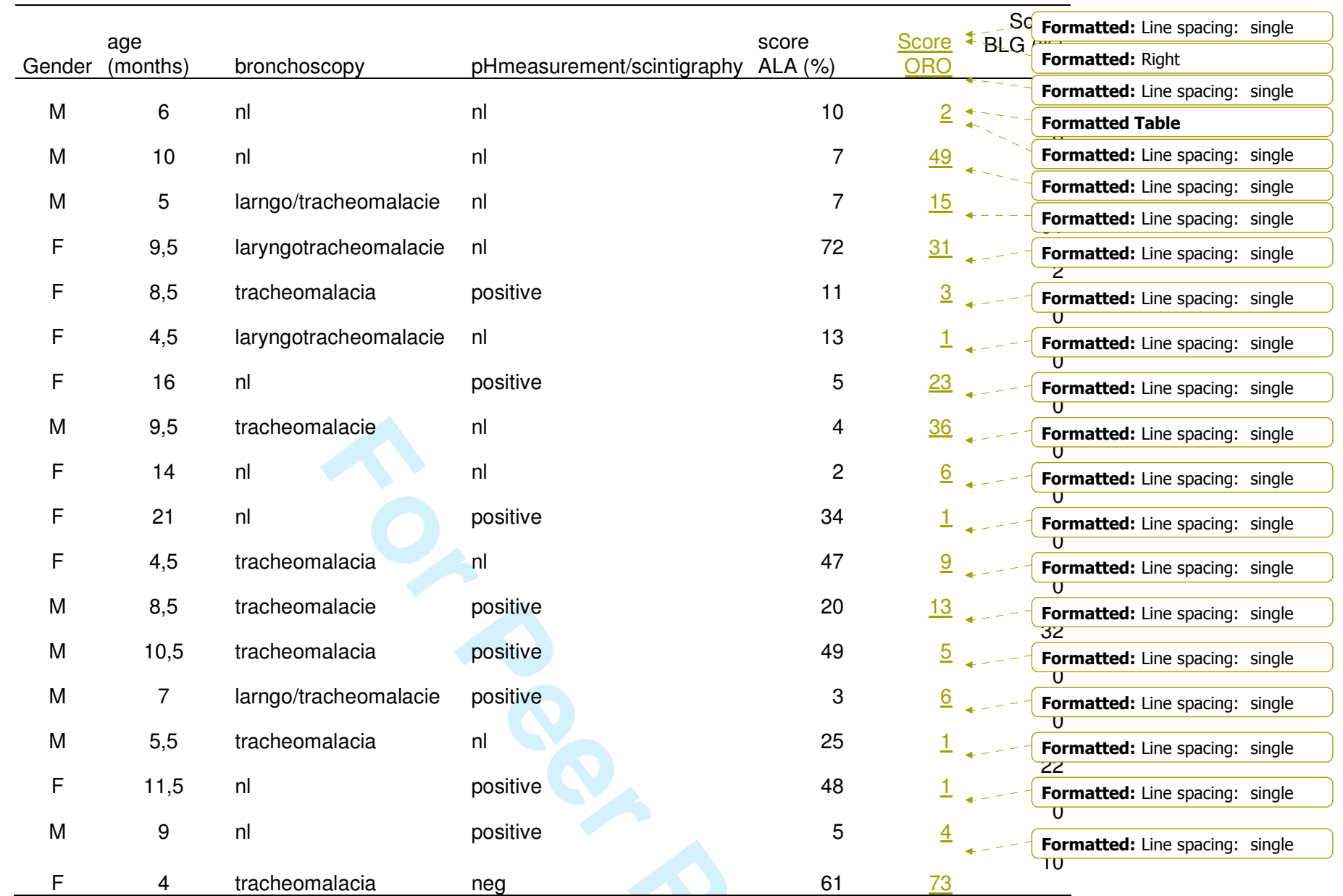


Fig 2: Concomitant findings in positive staining infants

8/18: positive GER test Positive staining

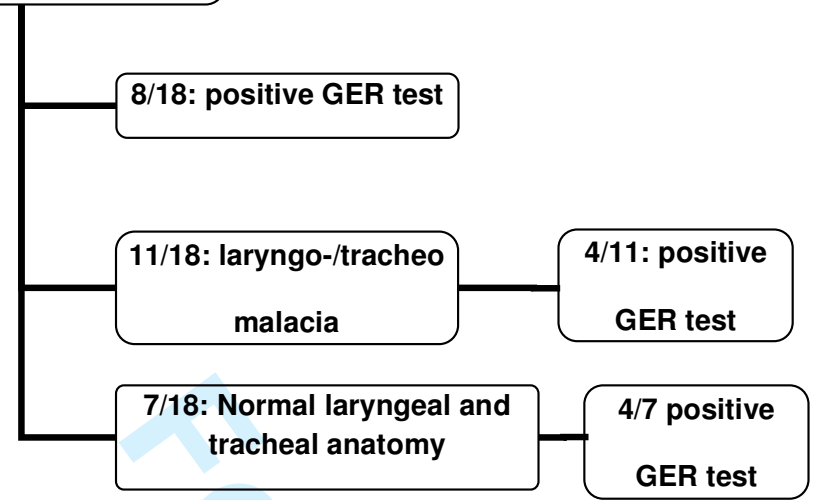


Fig. 3: A)

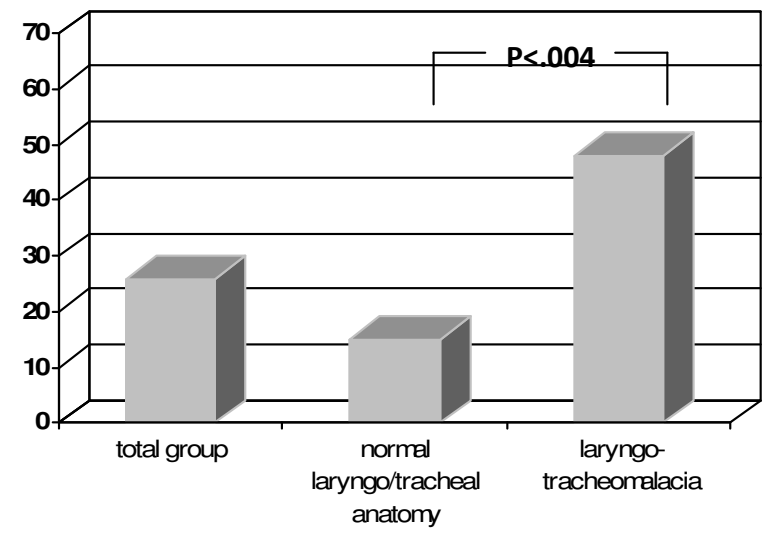

\% positively staining macrophages

C)

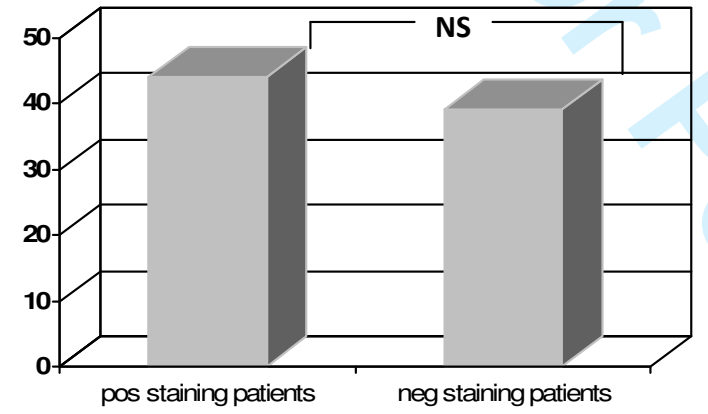

$\%$ pos GER tests

E)

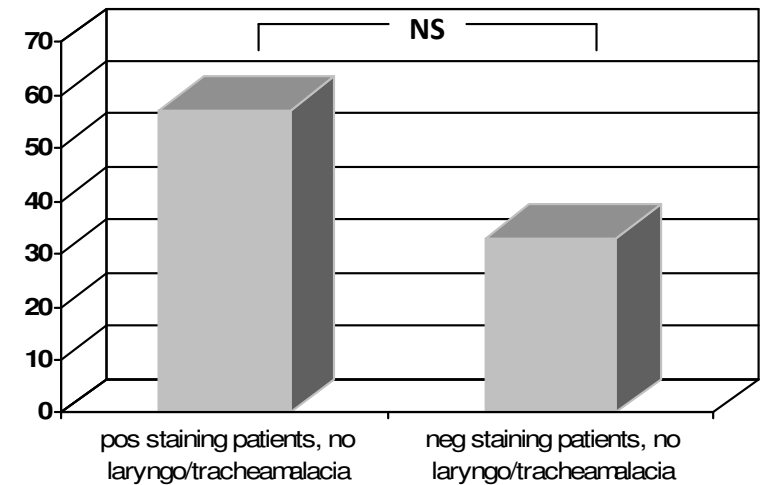

\% positive GER tests

pos staining patients, no neg staining patients, no

laryngo/tracheamalacia laryngo/tracheamelacia
B)

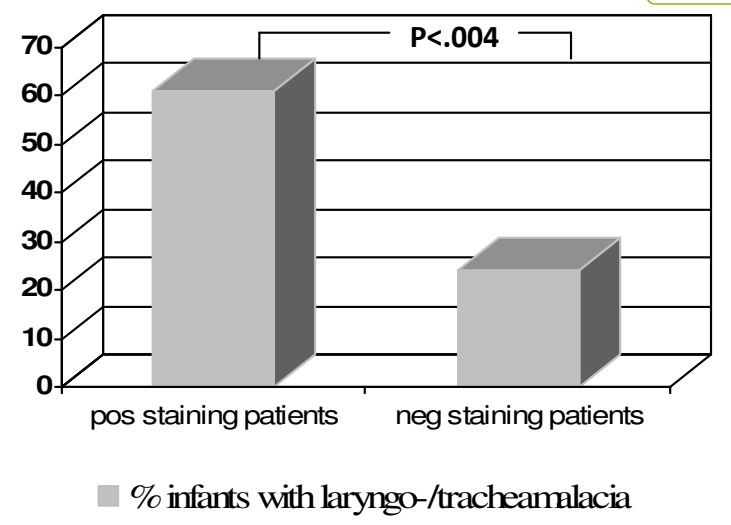

D)

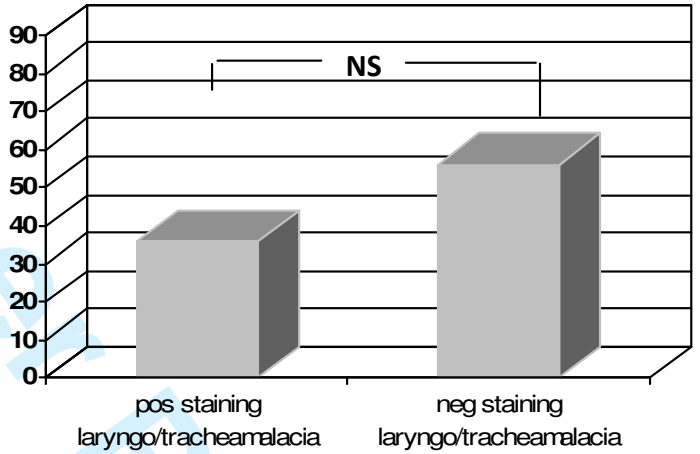

$\%$ positive GER tests
Formatted: Font: Bold

Formatted: Font: Bold
Formatted: Font: Bold

Formatted: Font: Bold

Formatted: Font: Bold 
Fig 4:

Correlation between Oil red O score and positive ALA score

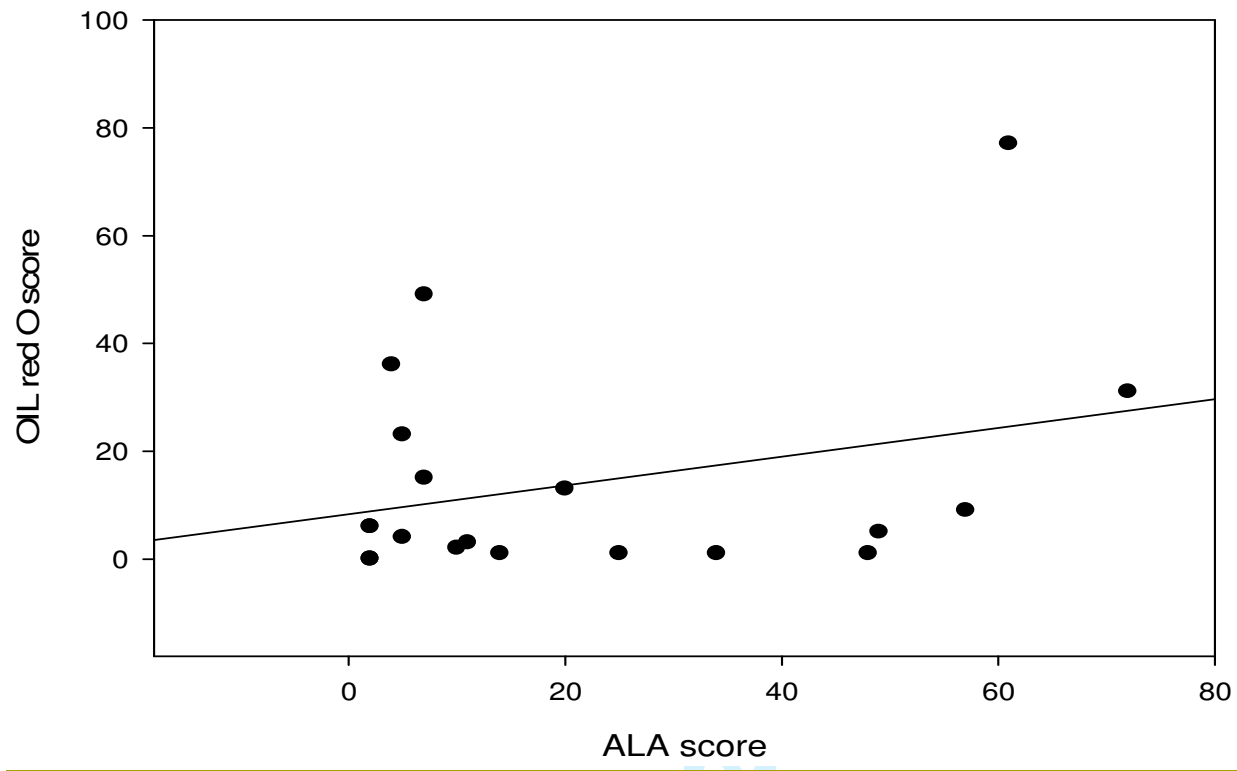

Oil-Red-O score : absolute numbers

Formatted: English (U.K.)

Formatted: Line spacing: single

ALA ( $\alpha$-lactalbumin): percentage

Formatted: Font: Not Bold

Formatted: English (U.K.) 


\section{REFERENCES}

1. Bauer ML, Figueroa-Colon R, Georgeson K, Young DW. Chronic pulmonary aspiration in children. Southern Medical Journal, July 2003; Vol. 86, No. 7

2. Knauer-Fischer S, Ratjen F. Lipid-laden macrophages in bronchoalveolar lavage fluid as a marker for pulmonary aspiration. Pediatr Pulmonol. 1999;27:419-422

3. Bauer ML, Lyrene RK. Chronic aspiration in children: evaluation of the lipid-laden macrophage index. Pediatr Pulmonol. 1999;28:94-100

4. Ahrens P, Noll C, Kitz R, Willigens P, Zielen S, Hofmann D. Lipid-laden alveolar macrophage (LLAM): a useful marker of silent aspiration in children. Pediatr Pulmonol. 1999;28:83-88

5. Kazachkov M, Muhlenbach M, Livasy C, Noah T. Lipid laden macrophages indices and inflammation in BAL fluid of children with respiratory symptoms. ERJ 2001; 18: 790-795.

6. Sacco O, Fregonese B, Silvestri M, Sabatini F, Mattioli G, Rossi GA. Bronchoalveolar lavage and esophageal $\mathrm{pH}$ monitoring data in children with "difficult to treat" respiratory symptoms. Pediatr Pulmonol. 2000;30:313-319

7. Sheikh S, Stephen T, Howell L, Eid N. Gastroesophageal reflux in infants with wheezing. Pediatr Pulmonol. 1999;28:181-186

8. Vijayaratnam V, Lin CH, Simpson P, Tolia V. Lack of significant proximal esophageal acid reflux in infants presenting with respiratory symptoms. Pediatr Pulmonol 1999;27:231-235

9. Medula F, Guidi R, Tancredi G, Quantrucci S, Ratjen F, Bottero S, Vestiti K, Francalanci P, Cutrera R. Microapiration in infants with laryngomalacia. Laryngoscope 2004; 114: 1592-96

10. Dickson JM, Richter GT, Meinzen-Derr J, Rutter MJ, Thompson JM. Secondary airway lesions in children with laryngomalacia. Ann Oto Rhinol Laryngol 2009; 118: 37-43.

11. Yalcin E, Dogru D, Ozcelik U, Kiper N, Aslan AT, Gözaçan A. Tracheomalacia and bronchomalacia in 34 children: clinical and radiological profiles and associations with other diseases. Clin Ped 2005; 44: 777-81

12. Elidemir O, Fan LL, Colasurdo GN. A novel diagnostic method for pulmonary aspiration in a murine model: immunocytochemical staining of milk proteins in alveolar macrophages. Am J Respir Crit Care Med. 2000; 161:622-626

13. Miller J, Colasurdo GN, Khan AM, Jajoo C, Patel TJ, Fan LL, Elidemir O. Immunocytochemical detection of milk proteins in tracheal aspirates of ventilated infants: a pilot study. Pediatric Pulmonol. 2002;34:369374

14. Farrell S, McMaster C, Gibson D, Shields MD, McCallion WA. Pepsin in bronchoalveolar lavage fluid: a specific and sensitive method of diagnosing gastro-oesophageal reflux-related pulmonary aspiration. Journal of Pediatric Surgery 2006;41:289-293

15. Colombo JL, Hallberg TK. Recurrent aspiration in children: lipid-laden alveolar macrophage quantitation. Pediatr Pulmonol 1987:3:86-89

16. Vandenplas Y. Oesophageal $\mathrm{pH}$ monitoring for gastroesophagealreflux in infants and children. Chichester: John Wiley; 1992.

17. Pediatric GE reflux clinical practice guidelines. J. Pediatr Gastroenterol Nutr, Vol. 32, Suppl. 2, 2001

18. Piepsz. Recent advances in Pediatric Nuclear medicine. Sem Nucl Med 1995; 25: 165-182

19. Ravelli AM, Panarotto MB, Verdoni L, Consolati V, Bolognini S. Pulmonary aspiration shown by scintigraphy in gastroesophageal reflux-related respiratory disease. Chest 2006;130:1520-1526

20. Gorenstein A, Levine A, Boaz M, Mandelberg A, Serour F. Severity of acid gastroesophageal reflux assessed by pH metry: is it associated with respiratory disease? Pediatr Pulmonol. 2003;36:330-334

21. Bobin S, Attal P. Laryngotracheal manifestations of gastroesophagael reflux in children. Pediatr Pulmonol. 1999, suppl 18:73-75 
Concomitant findings in positive staining infants 254x190mm (96 x 96 DPI)

John Wiley \& Sons, Inc. 


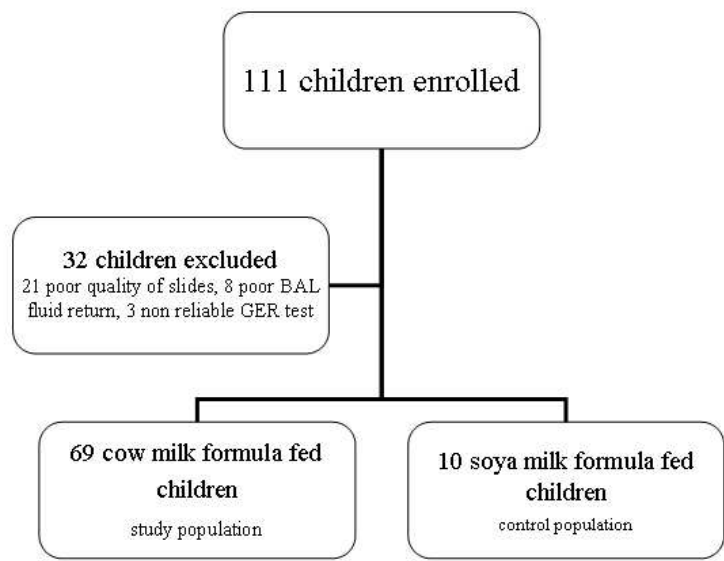

Study population 254×190mm (96 x 96 DPI)

John Wiley \& Sons, Inc. 
Oil-Red-O score : absolute numbers ALA (a-lactalbumin): percentage 254x190mm (96 x 96 DPI)

John Wiley \& Sons, Inc. 
Fig 3:
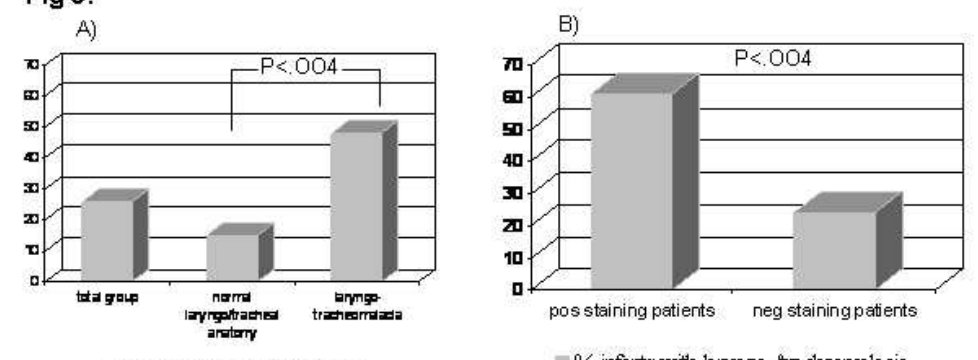

In \% positively staimingmacrophages

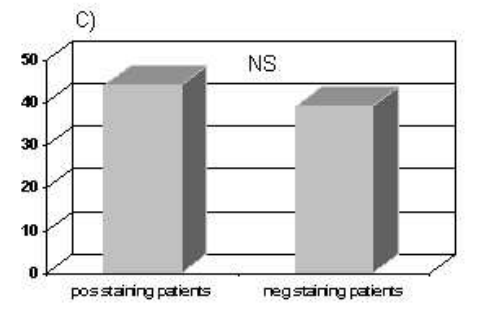

in \%os GrRtests 
Table 1: Characteristics of positively staining children (ALA: $\alpha$-lactalbumin, BLG: $\beta$ lactoglobulin, ORO: Oil-Red-O, nl: normal)

\begin{tabular}{|c|c|c|c|c|c|c|}
\hline Gender & $\begin{array}{l}\text { age } \\
\text { (months) }\end{array}$ & bronchoscopy & pHmeasurement/scintigraphy & $\begin{array}{l}\text { score } \\
\text { ALA (\%) }\end{array}$ & $\begin{array}{r}\text { Score } \\
\text { ORO }\end{array}$ & $\begin{array}{r}\text { Score } \\
\text { BLG (\%) }\end{array}$ \\
\hline$M$ & 6 & $\mathrm{nl}$ & $\mathrm{nl}$ & 10 & 2 & 13 \\
\hline M & 10 & $\mathrm{nl}$ & $\mathrm{nl}$ & 7 & 49 & 0 \\
\hline M & 5 & larngo/tracheomalacie & $\mathrm{nl}$ & 7 & 15 & 0 \\
\hline $\mathrm{F}$ & 9,5 & laryngotracheomalacie & $\mathrm{nl}$ & 72 & 31 & 51 \\
\hline $\mathrm{F}$ & 8,5 & tracheomalacia & positive & 11 & 3 & 2 \\
\hline $\mathrm{F}$ & 4,5 & laryngotracheomalacie & $\mathrm{nl}$ & 13 & 1 & 0 \\
\hline $\mathrm{F}$ & 16 & $\mathrm{nl}$ & positive & 5 & 23 & 0 \\
\hline M & 9,5 & tracheomalacie & $\mathrm{nl}$ & 4 & 36 & 0 \\
\hline $\mathrm{F}$ & 14 & $r>$ & $\mathrm{nl}$ & 2 & 6 & 0 \\
\hline $\mathrm{F}$ & 21 & $\mathrm{nl}$ & positive & 34 & 1 & 0 \\
\hline $\mathrm{F}$ & 4,5 & tracheomalacia & $\mathrm{nl}$ & 47 & 9 & 0 \\
\hline$M$ & 8,5 & tracheomalacie & positive & 20 & 13 & 0 \\
\hline M & 10,5 & tracheomalacia & positive & 49 & 5 & 32 \\
\hline$M$ & 7 & larngo/tracheomalacie & positive & 3 & 6 & 0 \\
\hline M & 5,5 & tracheomalacia & $\mathrm{nl}$ & 25 & 1 & 0 \\
\hline$F$ & 11,5 & $\mathrm{nl}$ & positive & 48 & 1 & 22 \\
\hline M & 9 & $\mathrm{nl}$ & positive & 5 & 4 & 0 \\
\hline $\mathrm{F}$ & 4 & tracheomalacia & neg & 61 & 73 & 10 \\
\hline
\end{tabular}

